Moment- equat i on met hods for cal cul at ing neocl assi cal transport coeffici ent $s$ i $n$ general tor oi dal pl asmas

\begin{tabular}{|l|l|}
\hline $\begin{array}{l}\mathrm{j} \text { our nal or } \\
\text { publ i cat i on } \mathrm{titl} \text { e }\end{array}$ & Physi cs of Pl asmas \\
\hline vol une & Vol . 15 \\
\hline page range & 042502-1- 042502-12 \\
\hline year & 2008 04-01 \\
\hline URL & ht t p: //hdl . handl e. net /10655/3838 \\
\hline
\end{tabular}




\title{
Moment-equation methods for calculating neoclassical transport coefficients in general toroidal plasmas
}

\author{
H. Sugama ${ }^{1,2}$ and S. Nishimura ${ }^{1}$ \\ ${ }^{1}$ National Institute for Fusion Science, Toki 509-5292, Japan \\ ${ }^{2}$ The Graduate University for Advanced Studies, Toki 509-5292, Japan
}

(Received 28 December 2007; accepted 4 March 2008; published online 11 April 2008)

\begin{abstract}
A detailed comparison is made between moment-equation methods presented by H. Sugama and S. Nishimura [Phys. Plasmas 9, 4637 (2002)] and by M. Taguchi [Phys. Fluids B 4, 3638 (1992)] for calculating neoclassical transport coefficients in general toroidal plasmas including nonsymmetric systems. It is shown that these methods can be derived from the drift kinetic equation with the same collision model used for correctly taking account of collisional momentum conservation. In both methods, the Laguerre polynomials of the energy variable are employed to expand the guiding-center distribution function and to obtain the moment equations, by which the radial neoclassical transport fluxes and the parallel flows are related to the thermodynamic forces. The methods are given here in the forms applicable for an arbitrary truncation number of the Laguerre-polynomial expansion so that their accuracies can be improved by increasing the truncation number. Differences between results from the two methods appear when the Laguerre-polynomial expansion is truncated up to a finite order because different weight functions are used in them to derive the moment equations. At each order of the truncation, the neoclassical transport coefficients obtained from the Sugama-Nishimura method show the Onsager symmetry and satisfy the ambipolar-diffusion condition intrinsically for symmetric systems. Also, numerical examples are given to show how the transport coefficients converge with the truncation number increased for the two methods. (C) 2008 American Institute of Physics. [DOI: 10.1063/1.2902012]
\end{abstract}

\section{INTRODUCTION}

Neoclassical transport is an important factor to determine plasma confinement, especially in nonaxisymmetric toroidal systems such as heliotrons and stellarators. ${ }^{1}$ For example, neoclassical transport fluxes due to particles trapped in helical ripples are expected to be significantly large for high temperature and play a key role in determining the radial electric field under the ambipolar-diffusion condition. ${ }^{2}$ Also, quasisymmetric toroidal systems are attracting much attention as an advanced concept, in which configurations are optimized to nearly suppress the neoclassical ripple transport and the neoclassical viscosity against flows in the direction of quasisymmetry. ${ }^{3-7}$ Furthermore, positive correlations between neoclassical and anomalous transport through generation of zonal flows in helical plasmas have been argued. ${ }^{8-12}$ In addition, even in tokamak experiments, the effects of the neoclassical toroidal viscosity due to the broken axisymmetry on the toroidal plasma rotation have recently been studied in relation to the stability of the resistive wall mode. ${ }^{13}$ Thus, it is required to do accurate and fast calculations of neoclassical transport coefficients, by which radial fluxes and tangential flows to flux surfaces are related to thermodynamic forces.

There are two methods known as moment-equation approaches to calculate the neoclassical transport coefficients in general toroidal systems with no symmetry. One is presented by Sugama and Nishimura ${ }^{14}$ and the other by Taguchi. ${ }^{15}$ Both methods show how to accurately take account of collisional momentum conservation in multispecies plasmas for obtaining the transport coefficients from an out- put of commonly used numerical codes such as the Drift Kinetic Equation Solver (DKES), ${ }^{16,17}$ in which the pitchangle-scattering collision model is used. These methods are useful for determining profiles of the neoclassical radial fluxes, tangential flows, and radial electric fields in various types of nonaxisymmetric toroidal systems as demonstrated in Refs. 18 and 19 while it is still important to address the theoretical relation between the methods as well as their accuracies from the viewpoint of practical application. ${ }^{20,21}$ For this purpose, a detailed comparison between the two methods is made in the present paper. Also, the methods are written here for an arbitrary truncation number of the Laguerrepolynomial expansion of the guiding-center distribution function in order that one can increase the truncation number to improve the accuracies. This is contrast to the original papers by Sugama and Nishimura and by Taguchi, where the methods are explicitly shown only for the case of retaining the first two terms in the expansion.

We find in Sec. II how the Sugama-Nishimura and Taguchi methods are derived from the drift kinetic equation including the same collision model and where a cause of differences between them occurs. There, the two methods are compared with each other from the viewpoints of the intrinsic ambipolar diffusion in the symmetric limit and of the Onsager symmetry ${ }^{22-24}$ of the transport coefficients. Furthermore, their different results are illustrated by numerical examples in Sec. III, where the ion banana neoclassical transport for the axisymmetric case is considered in order to judge their applicabilities to quasisymmetric systems by checking the accuracy of the results in the symmetric limit. Finally, 
conclusions are given in Sec. IV and dimensionless coefficients used for calculating the ion banana transport are shown in the Appendix.

\section{DERIVATION OF TWO METHODS FOR CALCULATING NEOCLASSICAL TRANSPORT COEFFICIENTS}

In this section, two methods for calculating neoclassical transport coefficients are derived from the drift kinetic equations and differences between their results are described in detail.

\section{A. Basic equations}

We consider toroidal configurations with the magnetic field written in terms of the flux coordinates $(s, \theta, \zeta)$ as

$$
\begin{aligned}
\mathbf{B} & =\psi^{\prime} \nabla s \times \nabla \theta+\chi^{\prime} \nabla \zeta \times \nabla s \\
& =B_{s} \nabla s+B_{\theta} \nabla \theta+B_{\zeta} \nabla \zeta,
\end{aligned}
$$

where $\theta$ and $\zeta$ represent the poloidal and toroidal angles, respectively, and $s$ is an arbitrary label of a flux surface. The poloidal and toroidal fluxes are given by $2 \pi \chi(s)$ $=(2 \pi)^{-1} \int_{V(s)} d^{3} x \mathbf{B} \cdot \nabla \theta$ and $2 \pi \psi(s)=(2 \pi)^{-1} \int_{V(s)} d^{3} x \mathbf{B} \cdot \nabla \zeta$, respectively, where $V(s)$ is the volume enclosed by the flux surface with the label $s$. The derivative with respect to $s$ is denoted by ${ }^{\prime}=d / d s$ so that $\psi^{\prime}=d \psi / d s$ and $\chi^{\prime}=d \chi / d s$. The covariant radial, poloidal, and toroidal components of the magnetic field $\mathbf{B}$ are written as $B_{s} \equiv \mathbf{B} \cdot \partial \mathbf{x} / \partial s \equiv \sqrt{g} \mathbf{B} \cdot(\nabla \theta$ $\times \nabla \zeta), \quad B_{\theta} \equiv \mathbf{B} \cdot \partial \mathbf{x} / \partial \theta \equiv \sqrt{g} \mathbf{B} \cdot(\nabla \zeta \times \nabla s)$, and $B_{\zeta} \equiv \mathbf{B} \cdot \partial \mathbf{x} / \partial \zeta$ $\equiv \sqrt{g} \mathbf{B} \cdot(\nabla s \times \nabla \theta), \quad$ respectively, where $\quad \sqrt{g} \equiv[\nabla s \cdot(\nabla \theta$ $\times \nabla \zeta)]^{-1}$ represents the Jacobian for the coordinates $(s, \theta, \zeta)$. Here, we may regard $(s, \theta, \zeta)$ as either Boozer ${ }^{25}$ coordinates, Hamada $^{26}$ coordinates, or arbitrary other flux coordinates.

The equilibrium distribution function for the particle species $a$ with the mass $m_{a}$ and the charge $e_{a}$ is given in the local Maxwellian form $f_{a M} \equiv \pi^{-3 / 2} n_{a} v_{T a}^{-3} \exp \left(-x_{a}^{2}\right)$, where $v_{T a} \equiv\left(2 T_{a} / m_{a}\right)^{1 / 2}$ denotes the thermal velocity, and $x_{a}$ $\equiv v / v_{T a}$ represents the normalized velocity. The equilibrium density $n_{a}$ and the temperature $T_{a}$ are flux surface functions independent of $\theta$ and $\zeta$. The deviation $f_{a 1}$ from the local Maxwellian is determined by the linearized drift kinetic equation,

$$
\begin{aligned}
V f_{a 1}-C_{a}\left(f_{a 1}\right)= & \frac{1}{T_{a}} f_{a M}\left(-\sigma_{1}^{+}\left[X_{a 1}+X_{a 2}\left(x_{a}^{2}-\frac{5}{2}\right)\right]\right. \\
& \left.+e_{a} \frac{B}{\left\langle B^{2}\right\rangle^{1 / 2}} v \xi X_{E}\right) .
\end{aligned}
$$

Here, $f_{a 1}$ is independent of the gyrophase and regarded as a function of the phase-space variables $(\mathbf{x}, v, \xi)$, where $\mathbf{x}$ is the position of the particle's guiding-center and $\xi$ is defined by $\xi \equiv v_{\|} / v$ with $v_{\|} \equiv \mathbf{v} \cdot \mathbf{b}$ and $\mathbf{b} \equiv \mathbf{B} / B$. Then, the operator $V$ on the left-hand side of Eq. (2) is defined by

$$
\begin{aligned}
& V \equiv V_{\|}+V_{E}, \\
& V_{\|} \equiv v \xi \mathbf{b} \cdot \nabla-\frac{1}{2} v\left(1-\xi^{2}\right)(\mathbf{b} \cdot \nabla \ln B) \frac{\partial}{\partial \xi}, \\
& V_{E} \equiv \frac{c E_{s}}{\left\langle B^{2}\right\rangle} \nabla s \times \mathbf{B} \cdot \nabla .
\end{aligned}
$$

The above $\mathbf{E} \times \mathbf{B}$ drift operator $V_{E}$ associated with the radial electric field $E_{s}=-\partial \Phi / \partial s$ ( $\Phi$ : the electrostatic potential) has the same form as employed in the DKES ${ }^{16,17}$ and by Taguchi, ${ }^{15}$ in which the compressional part of the $\mathbf{E} \times \mathbf{B}$ drift velocity is removed. The thermodynamic forces $X_{a 1}, X_{a 2}$, and $X_{E}$ on the right-hand side of Eq. (2) are defined by

$$
\begin{aligned}
& X_{a 1} \equiv-\frac{1}{n_{a}} \frac{\partial p_{a}}{\partial s}-e_{a} \frac{\partial \Phi}{\partial s}, \\
& X_{a 2} \equiv-\frac{\partial T_{a}}{\partial s}, \\
& X_{E} \equiv\left\langle B E_{\|}\right\rangle /\left\langle B^{2}\right\rangle^{1 / 2},
\end{aligned}
$$

respectively, where $p_{a} \equiv n_{a} T_{a}$ is the pressure, $E_{\|} \equiv \mathbf{E} \cdot \mathbf{b}$ the parallel electric field, and $\langle\cdots\rangle \equiv \oint d \theta \oint d \zeta \sqrt{g} \cdots / V^{\prime}$ with $V^{\prime}$ $\equiv \oint d \theta \oint d \zeta \sqrt{g}$ represents the flux surface average.

Now, it is convenient to consider the expansion of an arbitrary function $F(\mathbf{x}, v, \xi)$ by the Legendre polynomials $P_{l}(\xi)\left[P_{0}(\xi)=1, P_{1}(\xi)=\xi, P_{2}(\xi)=\frac{3}{2} \xi^{2}-\frac{1}{2}, \cdots\right]$ as

$$
\begin{aligned}
& F(\mathbf{x}, v, \xi)=\sum_{l=0}^{\infty} F^{(l)}(\mathbf{x}, v, \xi) \\
& F^{(l)}(\mathbf{x}, v, \xi)=P_{l}(\xi) \frac{2 l+1}{2} \int_{-1}^{1} d \eta P_{l}(\eta) F(\mathbf{x}, v, \eta) .
\end{aligned}
$$

Then, for the collision term $C_{a}\left(f_{a 1}\right)$ on the left-hand side of Eq. (2), we use the collision operator defined by

$$
\begin{aligned}
C_{a}\left(f_{a 1}\right)= & \sum_{b}\left[C_{a b}\left(f_{a 1}^{(l=1)}, f_{b M}\right)+C_{a b}\left(f_{a M}, f_{b 1}^{(l=1)}\right)\right] \\
& +\nu_{D}^{a} \mathcal{L}\left(f_{a 1}-f_{a 1}^{(l=1)}\right),
\end{aligned}
$$

where $C_{a b}$ is the Landau operator ${ }^{27}$ for Coulomb collisions between species $a$ and $b$ and the pitch-angle-scattering collision operator is defined by

$$
\nu_{D}^{a} \mathcal{L} \equiv \frac{\nu_{D}^{a}}{2} \frac{\partial}{\partial \xi}\left(1-\xi^{2}\right) \frac{\partial}{\partial \xi} .
$$

The energy-dependent collision frequency $\nu_{D}^{a}$ in Eq. (7) is given $b^{28}$

$$
\nu_{D}^{a} \equiv \sum_{b} \frac{3 \sqrt{\pi}}{4} \tau_{a b}^{-1} x_{a}^{-3} \mathcal{H}\left(x_{b}\right),
$$

where $(3 \sqrt{\pi} / 4) \tau_{a b}^{-1} \equiv 4 \pi n_{b} e_{a}^{2} e_{b}^{2} \ln \Lambda /\left(m_{a}^{2} v_{T a}^{3}\right)(\ln \Lambda$ : the Coulomb logarithm) and $\mathcal{H}(x) \equiv\left[\left(2 x^{2}-1\right) \Phi(x)+x \Phi^{\prime}(x)\right] /\left(2 x^{2}\right)$ $\left[\Phi(x) \equiv 2 \pi^{-1 / 2} \int_{0}^{x} \exp \left(-t^{2}\right) d t\right.$ : the error function]. The collision model shown in Eq. (6) is equivalent to those in Refs. 14 and 15. When using the collision operator shown in Eq. 
(6), the $l=0$ component $f_{a 1}^{(l=0)}$ never contributes to the collision term, $C_{a}\left(f_{a 1}^{(l=0)}\right)=0$. However, for the rigorous linearized collision operator defined by $C_{a}\left(f_{a 1}\right)=\Sigma_{b}\left[C_{a b}\left(f_{a 1}, f_{b M}\right)\right.$ $\left.+C_{a b}\left(f_{a M}, f_{b 1}\right)\right]$ with the Landau operator $C_{a b}, C_{a}\left(f_{a 1}^{(l=0)}\right)$ does not vanish generally but it vanishes only for the distribution function in the form of $f_{a 1}^{(l=0)}=F_{a M}\left[n_{a 1} / n_{a}+\left(x_{a}^{2}-3 / 2\right) T_{a 1}\right]$, where $n_{a 1}$ and $T_{a 1}$ represent the perturbations of the density and temperature, respectively. ${ }^{27}$ Since relative effects of the rigorous $l=0$ collision term $C_{a}\left(f_{a 1}^{(l=0)}\right)$ are weakened with decreasing collisionality, the collision model in Eq. (6) gives a better approximation in lower-collisional regimes. In order to correctly reproduce the viscosity coefficients for the PfirschSchlüter regime, we should replace $\nu_{D}^{a}$ with $\nu_{T}^{a} / 3$ in Eq. (7) for that collisional region, ${ }^{28}$ where $\nu_{T}^{a}$ is given by $\nu_{T}^{a} \equiv 3 \nu_{D}^{a}$ $+\nu_{E}^{a} \equiv(3 \sqrt{\pi} / 4) \Sigma_{b} \tau_{a b}^{-1}\left[\left\{\Phi\left(x_{b}\right)-3 G\left(x_{b}\right)\right\} / x_{a}^{3}+4\left(T_{a} / T_{b}\right)(1\right.$ $\left.\left.+m_{b} / m_{a}\right) G\left(x_{b}\right) / x_{a}\right]$ with $G(x) \equiv\left[\Phi(x)-x \Phi^{\prime}(x)\right] /\left(2 x^{2}\right)$.

The source functions $\sigma_{1}^{+}$in Eq. (2) and $\sigma_{3}^{+}$used later in Eq. (15) are defined by

$$
\begin{aligned}
\sigma_{1}^{+} & \equiv-\mathbf{v}_{d a} \cdot \nabla s \\
& =-\frac{2 v^{2}}{3 \Omega_{a}}\left[1+\frac{1}{2} P_{2}(\xi)\right] \mathbf{b} \times \nabla \ln B \cdot \nabla s, \\
\sigma_{3}^{+} & \equiv V\left(\frac{B}{\nu_{D}^{a}} v \xi\right),
\end{aligned}
$$

where $\mathbf{v}_{d a} \equiv\left(c / e_{a} B\right) \mathbf{b} \times\left(m_{a} v_{\|}^{2} \mathbf{b} \cdot \nabla \mathbf{b}+\mu \nabla B+e_{a} \nabla \Phi\right)$ is the guiding-center drift velocity and $\Omega_{a} \equiv e_{a} B /\left(m_{a} c\right)$ is the gyrofrequency. We note that the definition of $\sigma_{3}^{+}$in Eq. (9) follows from Rij and Hirshman ${ }^{17}$ and differs from that in Ref. 16.

We now define the inner product $(\alpha, \beta)$ for arbitrary functions $\alpha(\theta, \zeta, \xi)$ and $\beta(\theta, \zeta, \xi)$ by using the $\xi$ integral and the flux surface average as

$$
(\alpha, \beta) \equiv \frac{1}{2} \int_{-1}^{1} d \xi\langle\alpha \beta\rangle
$$

Then, we find that, with respect to this inner product, the operators $V$ and $\mathcal{L}$ are found to be antisymmetric and symmetric, respectively,

$$
(V \alpha, \beta)=-(\alpha, V \beta), \quad(\mathcal{L} \alpha, \beta)=(\alpha, \mathcal{L} \beta) .
$$

We should note that the energy integral (or integral in $v$ ) is not included in the inner product defined by Eq. (10) in contrast to that in Refs. 16 and 17 and that the energy integral is not necessary for the symmetry properties in Eq. (11) to hold. Also, it is useful to define the operators $\mathcal{P}_{\xi E}$ and $\mathcal{P}_{\theta \zeta}$ which act on an arbitrary function $f\left(\theta, \zeta, \xi, E_{s}\right)$ by

$$
\begin{aligned}
& \left(\mathcal{P}_{\xi E} f\right)\left(\theta, \zeta, \xi, E_{s}\right) \equiv f\left(\theta, \zeta,-\xi,-E_{s}\right), \\
& \left(\mathcal{P}_{\theta \xi} f\right)\left(\theta, \zeta, \xi, E_{s}\right) \equiv f\left(-\theta,-\zeta, \xi, E_{s}\right),
\end{aligned}
$$

where $\mathcal{P}_{\xi E}$ represents the time-reversal operation. ${ }^{17}$ Then, we obtain the following relations:

$$
\begin{aligned}
& \mathcal{P}_{\xi E} V=-V \mathcal{P}_{\xi E}, \quad \mathcal{P}_{\xi E} \mathcal{L}=\mathcal{L} \mathcal{P}_{\xi E}, \\
& \mathcal{P}_{\xi E} \sigma_{1}^{+}=\sigma_{1}^{+}, \quad \mathcal{P}_{\xi E} \sigma_{3}^{+}=\sigma_{3}^{+} .
\end{aligned}
$$

Similarly, for toroidal systems in which the stellarator symmetry ${ }^{17}$ is satisfied, we can derive the following relations:

$$
\begin{aligned}
& \mathcal{P}_{\theta \zeta} V=-V \mathcal{P}_{\theta \zeta}, \quad \mathcal{P}_{\theta \zeta} \mathcal{L}=\mathcal{L} \mathcal{P}_{\theta \zeta}, \\
& \mathcal{P}_{\theta \zeta} \sigma_{1}^{+}=-\sigma_{1}^{+}, \quad \mathcal{P}_{\theta \zeta} \sigma_{3}^{+}=-\sigma_{3}^{+}, \\
& \left(\mathcal{P}_{\theta \zeta} F, G\right)=\left(F, \mathcal{P}_{\theta \zeta} G\right) .
\end{aligned}
$$

For simplicity, we hereafter assume the stellarator symmetry to hold, although the results in the present work can be straightforwardly generalized to systems without the stellarator symmetry. Then, we see from Eqs. (13) and (14) that the two operations $\mathcal{P}_{\xi E}$ and $-\mathcal{P}_{\theta \zeta}$ have the same properties with respect to $V, \mathcal{L}$, and $\sigma_{j}^{+}(j=1,3)$. Multiplying -1 by $\mathcal{P}_{\theta \zeta} V$ $=-V \mathcal{P}_{\theta \zeta}$ and $\mathcal{P}_{\theta \zeta} \mathcal{L}=\mathcal{L} \mathcal{P}_{\theta \zeta}$ in Eq. (14), $\left(-\mathcal{P}_{\theta \zeta}\right) V=-V\left(-\mathcal{P}_{\theta \zeta}\right)$ and $\left(-\mathcal{P}_{\theta \zeta}\right) \mathcal{L}=\mathcal{L}\left(-\mathcal{P}_{\theta \zeta}\right)$ are immediately obtained, respectively, which shows that $-\mathcal{P}_{\theta \zeta}$ has the same properties as $\mathcal{P}_{\xi E}$ in Eq. (13) with respect to $V$ and $\mathcal{L}$.

In Ref. 17, the response functions $F_{j}^{+}$and $F_{j}^{-}(j=1,3)$ associated with the source terms $\sigma_{j}^{+}(j=1,3)$ are defined by

$$
\begin{aligned}
& V F_{j}^{-}-\nu_{D}^{a} \mathcal{L} F_{j}^{+}=\sigma_{j}^{+}, \\
& V F_{j}^{+}-\nu_{D}^{a} \mathcal{L} F_{j}^{-}=0 \quad(j=1,3)
\end{aligned}
$$

and the monoenergetic transport coefficients $D_{j k}(K)(j, k$ $=1,3$ ) are defined by

$$
D_{j k}(K) \equiv\left(\sigma_{j}^{+}, F_{k}^{+}\right)+\left(\sigma_{j}^{+}, F_{k}^{-}\right) \quad(j, k=1,3),
$$

where $K \equiv x_{a}^{2} \equiv m_{a} v^{2} / 2 T_{a}$ represents the normalized kinetic energy. We find from Eqs. (13)-(16) that

$$
\begin{aligned}
& \mathcal{P}_{\xi E} F_{j}^{+}=-\mathcal{P}_{\theta \zeta} F_{j}^{+}=F_{j}^{+}, \\
& \mathcal{P}_{\xi E} F_{j}^{-}=-\mathcal{P}_{\theta \zeta} F_{j}^{-}=-F_{j}^{-}, \\
& \left(\sigma_{j}^{+}, F_{k}^{-}\right)=0,
\end{aligned}
$$

and that $D_{j k}(j, k=1,3)$ are even functions of $E_{s}$.

In Ref. 15 , other response functions $g_{a}^{(j)}(j=1,2)$ are defined by Taguchi as

$$
\begin{aligned}
& \left(V+\nu_{D}^{a} \mathcal{L}\right) g_{a}^{(1)}=f_{a M} B v \xi, \\
& \left(V+\nu_{D}^{a} \mathcal{L}\right) g_{a}^{(2)}=-f_{a M} \sigma_{1}^{+} \quad(j=1,3) .
\end{aligned}
$$

Comparing Eq. (15) with Eq. (18) and using Eqs. (9), (13), and $(14)$, we find that $g_{a}^{(j)}(j=1,2)$ can be expressed in terms of $F_{j}^{+}$and $F_{j}^{-}(j=1,3)$ as 


$$
\begin{aligned}
g_{a}^{(1)} & =f_{a M}\left[-\mathcal{P}_{\xi E}\left(F_{3}^{+}+F_{3}^{-}\right)-B v \xi / \nu_{D}^{a}\right] \\
& =f_{a M}\left[\mathcal{P}_{\theta \zeta}\left(F_{3}^{+}+F_{3}^{-}\right)-B v \xi / \nu_{D}^{a}\right], \\
g_{a}^{(2)} & =f_{a M} \mathcal{P}_{\xi E}\left(F_{1}^{+}+F_{1}^{-}\right) \\
& =-f_{a M} \mathcal{P}_{\theta \zeta}\left(F_{1}^{+}+F_{1}^{-}\right),
\end{aligned}
$$

which can be combined with Eq. (16) to derive

$$
\begin{aligned}
& \left(B v \xi, g_{a}^{(1)}\right)=f_{a M}\left[D_{33}(K)-\frac{v^{2}\left\langle B^{2}\right\rangle}{3 \nu_{D}^{a}}\right], \\
& \left(\sigma_{1}^{+}, g_{a}^{(1)}\right)=\left(B v \xi, g_{a}^{(2)}\right)=-f_{a M} D_{13}(K), \\
& \left(\sigma_{1}^{+}, g_{a}^{(2)}\right)=f_{a M} D_{11}(K) .
\end{aligned}
$$

Now, let us expand the $l=1$ Legendre component $f_{a 1}^{(l=1)}$ of the distribution function $f_{a 1}$ in terms of the Laguerre polynomials $L_{j}^{(3 / 2)}\left(x_{a}^{2}\right)\left[L_{0}^{(3 / 2)}\left(x_{a}^{2}\right)=1, L_{1}^{(3 / 2)}\left(x_{a}^{2}\right)=\frac{5}{2}-x_{a}^{2}, \cdots\right]$ as

$$
\begin{aligned}
f_{a 1}^{(l=1)} & =\frac{2}{v_{T a}} \xi x_{a} f_{a M}\left[u_{\| a}+\frac{2}{5} \frac{q_{\| a}}{p_{a}}\left(x_{a}^{2}-\frac{5}{2}\right)+\cdots\right] \\
& =\frac{2}{v_{T a}} \xi x_{a} f_{a M} \sum_{j=0}^{\infty} u_{\| a j} L_{j}^{(3 / 2)}\left(x_{a}^{2}\right),
\end{aligned}
$$

where

$$
\begin{aligned}
& u_{\| a j} \equiv \frac{c_{j}}{n_{a}} \int d^{3} v f_{a 1} v_{\|} L_{j}^{(3 / 2)}\left(x_{a}^{2}\right), \\
& c_{j} \equiv \frac{3 \cdot 2^{j} \cdot j !}{(2 j+3) ! !} .
\end{aligned}
$$

The coefficients of the first and second Laguerre polynomial components in Eq. (21) are given by $u_{\| a 0}=u_{\| a}$ and $u_{\| a 1}$ $=-(2 / 5)\left(q_{\| a} / p_{a}\right)$, where $u_{\| a} \equiv n_{a}^{-1} \int d^{3} v f_{a 1} v_{\|}$and $q_{\| a}$ $\equiv T_{a} \int d^{3} v f_{a 1} v_{\|}\left(x_{a}^{2}-\frac{5}{2}\right)$ represent the parallel fluid velocity and the parallel heat flow, respectively. Also, the $l=1$ Legendre component of the collision term in Eq. (6) is written as

$$
\begin{aligned}
& C_{a b}\left(f_{a 1}^{(l=1)}, f_{b M}\right)+C_{a b}\left(f_{a M}, f_{b 1}^{(l=1)}\right) \\
& \quad=\frac{1}{p_{a}} \xi v f_{a M}\left[F_{\| a 1}+\frac{2}{5} F_{\| a 2}\left(x_{a}^{2}-\frac{5}{2}\right)+\cdots\right] \\
& \quad=\frac{2}{v_{T a}} \xi x_{a} f_{a M} \sum_{j=0}^{\infty} C_{\| a j} L_{j}^{(3 / 2)}\left(x_{a}^{2}\right),
\end{aligned}
$$

where

$$
\begin{aligned}
C_{\| a j} \equiv & \frac{c_{j}}{n_{a}} \int d^{3} v v_{\|} L_{j}^{(3 / 2)}\left(x_{a}^{2}\right)\left[C_{a b}\left(f_{a 1}^{(l=1)}, f_{b M}\right)\right. \\
& \left.+C_{a b}\left(f_{a M}, f_{b 1}^{(l=1)}\right)\right] \\
= & \frac{c_{j}}{n_{a} m_{a}} \sum_{b} \sum_{k=0}^{\infty} l_{j+1, k+1}^{a b} u_{\| b k} .
\end{aligned}
$$

Here, the first two-order parallel friction forces are written as $F_{\| a 1}=n_{a} m_{a} C_{\| a 0}=\int d^{3} v C_{a}\left(f_{a 1}\right) m_{a} v_{\|} \quad$ and $\quad F_{\| a 2}=-\frac{5}{2} n_{a} m_{a} C_{\| a 1}$ $=\int d^{3} v C_{a}\left(f_{a 1}\right) m_{a} v_{\|}\left(x_{a}^{2}-\frac{5}{2}\right)$. The friction coefficients $l_{j+1, k+1}^{a b}$ are defined by ${ }^{28}$

$$
\begin{aligned}
l_{j+1, k+1}^{a b} \equiv & \int d^{3} v m_{a} v_{\|} L_{j}^{(3 / 2)}\left(x_{a}^{2}\right) \\
& \times\left[\delta_{a b} \sum_{b^{\prime}} C_{a b^{\prime}}\left(\frac{m_{a} v_{\|}}{T_{a}} L_{k}^{(3 / 2)}\left(x_{a}^{2}\right) f_{a M}, f_{b^{\prime} M}\right)\right. \\
& \left.+C_{a b}\left(f_{a M}, \frac{m_{b} v_{\|}}{T_{b}} L_{k}^{(3 / 2)}\left(x_{b}^{2}\right) f_{b M}\right)\right],
\end{aligned}
$$

where $\delta_{a b}$ denotes the Kronecker delta $\left(\delta_{a b}=1\right.$ for $a=b$ and $\delta_{a b}=0$ for $a \neq b$ ). The self-adjointness of the collision operator $C_{a b}$ and the momentum-conservation property $\Sigma_{a} F_{\| a 1}=0$ are written in terms of the friction coefficients as

$$
l_{j+1, k+1}^{a b}=l_{k+1, j+1}^{b a}, \quad \sum_{a} l_{1, k+1}^{a b}=0 .
$$

Averaging Eq. (18) with respect to $\xi$ yields

$$
\begin{aligned}
\mathbf{B} & \cdot \nabla\left(\frac{v}{B} \int_{-1}^{1} \frac{d \xi}{2} g_{a}^{(1)} \xi\right)+V_{E}\left(\int_{-1}^{1} \frac{d \xi}{2} g_{a}^{(1)}\right)=0, \\
\mathbf{B} & \cdot \nabla\left(\frac{v}{B} \int_{-1}^{1} \frac{d \xi}{2} g_{a}^{(2)} \xi\right)+V_{E}\left(\int_{-1}^{1} \frac{d \xi}{2} g_{a}^{(2)}\right) \\
= & f_{a M} \frac{2 v^{2}}{3 \Omega_{a}}(\mathbf{b} \times \nabla \ln B) \cdot \nabla s .
\end{aligned}
$$

Following Refs. 14 and 15 to neglect terms operated with $V_{E}$ in Eq. (27) [see remarks between Eqs. (E2) and (E3) in Appendix E of Ref. 14 and after Eq. (8) in Ref. 15], we obtain

$$
\begin{aligned}
& v \int_{-1}^{1} \frac{d \xi}{2} g_{a}^{(1)} \xi=\frac{B}{\left\langle B^{2}\right\rangle}\left(g_{a}^{(1)}, B v \xi\right), \\
& v \int_{-1}^{1} \frac{d \xi}{2} g_{a}^{(2)} \xi=\frac{B}{\left\langle B^{2}\right\rangle}\left(g_{a}^{(2)}, B v \xi\right)+f_{a M} \frac{v^{2}}{3} \frac{B \widetilde{U}}{\Omega_{a}},
\end{aligned}
$$

where $\tilde{U}$ is defined as a solution of

$$
\mathbf{B} \cdot \nabla\left(\frac{\tilde{U}}{B}\right)=\mathbf{B} \times \nabla s \cdot \nabla\left(\frac{1}{B^{2}}\right), \quad\langle B \tilde{U}\rangle=0 .
$$

From Eqs. (27) and (28), we can roughly estimate the relative effects of the neglected $V_{E}$ terms on Eq. (28) as small as of the order of $c|\nabla \Phi| /\left(v_{T a} B\right) \sim \rho_{T a} / L \ll 1$, where $\nabla \Phi \sim \Phi / L$, $e_{a} \Phi \sim T_{a}$, and $\rho_{T a} \equiv v_{T a} /\left|\Omega_{a}\right| \ll L$ are used. Similarly, averaging Eq. (2) with respect to $\xi$ and taking its Laguerre component of the order $j(=0,1,2, \cdots)$ lead to

$$
\begin{aligned}
& u_{\| a 0} \equiv u_{\| a}=\frac{B}{\left\langle B^{2}\right\rangle}\left\langle B u_{\| a}\right\rangle+\frac{c X_{a 1}}{e_{a}} \tilde{U}, \\
& -u_{\| a 1} \equiv \frac{2}{5 p_{a}} q_{\| a}=\frac{2}{5 p_{a}} \frac{B}{\left\langle B^{2}\right\rangle}\left\langle B q_{\| a}\right\rangle+\frac{c X_{a 2}}{e_{a}} \tilde{U}, \\
& u_{\| a j}=\frac{B}{\left\langle B^{2}\right\rangle}\left\langle B u_{\| a j}\right\rangle \quad(j \geqslant 2) .
\end{aligned}
$$


Now, we take inner products between $g_{a}^{(j)}(j=1,2)$ and Eq. (2) and use Eqs. (11) and (18) to derive

$$
\begin{aligned}
\left(g_{a}^{(1)}, \nu_{D}^{a} \mathcal{L} f_{a 1}-C_{a}\left(f_{a 1}\right)\right)-f_{a M}\left(B v \xi, f_{a 1}\right) & \\
= & \frac{1}{T_{a}} f_{a M}\left[-\left(g_{a}^{(1)}, \sigma_{1}^{+}\right)\left(X_{a 1}-X_{a 2} L_{1}^{(3 / 2)}(K)\right)\right. \\
& \left.+\left(g_{a}^{(1)}, B v \xi\right) \frac{e_{a} X_{E}}{\left\langle B^{2}\right\rangle^{1 / 2}}\right]
\end{aligned}
$$

and

$$
\begin{aligned}
& \left(g_{a}^{(2)}, \nu_{D}^{a} \mathcal{L} f_{a 1}-C_{a}\left(f_{a 1}\right)\right)+f_{a M}\left(\sigma_{1}^{+}, f_{a 1}\right) \\
& =\frac{1}{T_{a}} f_{a M}\left[-\left(g_{a}^{(2)}, \sigma_{1}^{+}\right)\left(X_{a 1}-X_{a 2} L_{1}^{(3 / 2)}(K)\right)\right. \\
& \left.\quad+\left(g_{a}^{(2)}, B v \xi\right) \frac{e_{a} X_{E}}{\left\langle B^{2}\right\rangle^{1 / 2}}\right] .
\end{aligned}
$$

Using Eqs. (20) and (24), we can rewrite Eq. (31) as

$$
\begin{aligned}
& -m_{a} \nu_{D}^{a}(K) D_{33}(K) \sum_{j=0}^{\infty} L_{j}^{(3 / 2)}(K)\left\langle B u_{\| a j}\right\rangle /\left\langle B^{2}\right\rangle \\
& -m_{a}\left[D_{33}(K)-\frac{2 T_{a} K\left\langle B^{2}\right\rangle}{3 m_{a} \nu_{D}^{a}(K)}\right] \sum_{j=0}^{\infty} c_{j} L_{j}^{(3 / 2)}(K) \\
& \quad \times \sum_{b} \sum_{k=0}^{\infty} \frac{l_{j+1, k+1}^{a b}}{n_{a} m_{a}}\left\langle B u_{\| b k}\right\rangle /\left\langle B^{2}\right\rangle \\
& =D_{13}(K)\left[X_{a 1}-X_{a 2} L_{1}^{(3 / 2)}(K)\right] \\
& +\left[D_{33}(K)-\frac{2 T_{a} K\left\langle B^{2}\right\rangle}{3 m_{a} \nu_{D}^{a}(K)}\right] \frac{e_{a} X_{E}}{\left\langle B^{2}\right\rangle^{1 / 2}}
\end{aligned}
$$

When we truncate the Laguerre-polynomial expansion up to the finite order of $j=j_{\max }$, we can derive the expressions that relate the averaged parallel flows, $\left\langle B u_{\| a 0}\right\rangle,\left\langle B u_{\| a 1}\right\rangle, \cdots$ $\left\langle B u_{\| a j_{\max }}\right\rangle(a=e, i, \cdots)$ to the thermodynamic forces $X_{a 1}, X_{a 2}$ $(a=e, i, \cdots)$, and $X_{E}$ from the moment equations given by multiplying Eq. (33) with appropriate weight functions of $K$ and taking the integral in $K$. As shown in the next subsections, the difference between the Sugama-Nishimura method and Taguchi's method results from using different weight functions to derive these moment equations.

Using Eqs. (20) and (24), we can rewrite Eq. (32) as a useful expression to derive the radial transport fluxes,

$$
\left(\mathbf{v}_{d a} \cdot \nabla s, f_{a 1}\right)=f_{a M}\left[\mathcal{F}_{a}^{\mathrm{PS}}(K)+\mathcal{F}_{a}^{\mathrm{bn}}(K)\right],
$$

where $\mathcal{F}_{a}^{\mathrm{PS}}(K)$ represents the monoenergetic contribution to the Pfirsch-Schlüter particle flux defined by

$$
\begin{aligned}
\mathcal{F}_{a}^{\mathrm{PS}}(K)= & -\frac{2}{3} \frac{K}{n_{a}}\left\langle\widetilde{U}^{2}\right\rangle \sum_{j=0}^{\infty} c_{j} L_{j}^{(3 / 2)}(K) \\
& \times \sum_{b} \frac{c^{2}}{e_{a} e_{b}}\left(l_{j+1,1}^{a b} X_{b 1}-l_{j+1,2}^{a b} X_{b 2}\right),
\end{aligned}
$$

and the other part $\mathcal{F}_{a}^{\mathrm{bn}}(K)$ of the monoenergetic particle flux is defined by

$$
\begin{aligned}
\mathcal{F}_{a}^{\mathrm{bn}}(K)= & \frac{m_{a}}{T_{a}} D_{13}(K) \sum_{j=0}^{\infty} L_{j}^{(3 / 2)}(K)\left[\nu_{D}^{a}(K) \frac{\left\langle B u_{\| a j}\right\rangle}{\left\langle B^{2}\right\rangle}\right. \\
& \left.+\frac{c_{j}}{n_{a} m_{a}} \sum_{b} \sum_{k=0}^{\infty} l_{j+1, k+1}^{a b} \frac{\left\langle B u_{\| b k}\right\rangle}{\left\langle B^{2}\right\rangle}\right] \\
& +\frac{1}{T_{a}}\left[D_{11}(K)-\frac{2 c^{2} m_{a} T_{a} K \nu_{D}^{a}(K)}{3 e_{a}^{2}}\left\langle\widetilde{U}^{2}\right\rangle\right] \\
& \times\left[X_{a 1}-X_{a 2} L_{1}^{(3 / 2)}(K)\right]+\frac{D_{13}(K)}{T_{a}} \frac{e_{a} X_{E}}{\left\langle B^{2}\right\rangle^{1 / 2}}
\end{aligned}
$$

The radial particle, heat, and other higher-order-moment fluxes can be obtained by multiplying Eq. (34) with $L_{j}^{(3 / 2)}(K)(j=0,1,2, \cdots)$ and taking the $K$ integrals. Defining the average operator $\{\cdots\}$ for an arbitrary function $A(K)$ by

$$
\{A(K)\} \equiv \frac{4}{3 \sqrt{\pi}} \int_{0}^{\infty} d K K^{3 / 2} e^{-K} A(K),
$$

we obtain the orthogonality relation

$$
\left\{L_{j}^{(3 / 2)}(K) L_{k}^{(3 / 2)}(K)\right\}=\frac{1}{c_{j}} \delta_{j k} \quad(j, k=0,1,2, \cdots) .
$$

Then, the Pfirsch-Schlüter (PS) particle and heat fluxes are immediately calculated from Eq. (35) as

$$
\begin{aligned}
\Gamma_{a j}^{\mathrm{PS}} & =n_{a} \frac{2}{\sqrt{\pi}} \int_{0}^{\infty} d K \sqrt{K} e^{-K} \mathcal{F}_{a}^{\mathrm{PS}}(K) L_{j}^{(3 / 2)}(K) \\
& =n_{a}\left\{\frac{3}{2} K^{-1} \mathcal{F}_{a}^{\mathrm{PS}}(K) L_{j}^{(3 / 2)}(K)\right\} \\
& =-\sum_{b} \frac{c^{2}}{e_{a} e_{b}}\left\langle\widetilde{U}^{2}\right\rangle\left(l_{j 1}^{a b} X_{b 1}-l_{j 2}^{a b} X_{b 2}\right) \quad(j=0,1,2, \cdots) .
\end{aligned}
$$

The Pfirsch-Schlüter particle and heat fluxes are given from Eq. (39) by $\Gamma_{a}^{\mathrm{PS}}=\Gamma_{a 0}^{\mathrm{PS}}$ and $q_{a}^{\mathrm{PS}} / T_{a}=-\Gamma_{a 1}^{\mathrm{PS}}$, respectively. Similarly, using Eq. (36), we obtain the radial neoclassical fluxes,

$$
\begin{aligned}
\Gamma_{a j}^{\mathrm{bn}} & \equiv n_{a} \frac{2}{\sqrt{\pi}} \int_{0}^{\infty} d K \sqrt{K} e^{-K} \mathcal{F}_{a}^{\mathrm{bn}}(K) L_{j}^{(3 / 2)}(K) \\
& =n_{a}\left\{\frac{3}{2} K^{-1} \mathcal{F}_{a}^{\mathrm{bn}}(K) L_{j}^{(3 / 2)}(K)\right\} \quad(j=0,1,2, \cdots),
\end{aligned}
$$

where the $j=0$ and 1 cases give the radial particle and heat fluxes, respectively, as $\Gamma_{a}^{\mathrm{bn}}=\Gamma_{a 0}^{\mathrm{bn}}$ and $q_{a}^{\mathrm{bn}} / T_{a}=-\Gamma_{a 1}^{\mathrm{bn}}$. Here, $\Gamma_{a j}^{\mathrm{bn}}$ can be written as the sum of the banana-plateau and the nonsymmetric parts. ${ }^{23,31}$ The banana-plateau part of the radial particle fluxes are intrinsically ambipolar and proportional to the neoclassical viscosity in the direction of the magnetic field while the neoclassical ripple diffusion is given by the nonsymmetric part that arises because the viscosity never vanishes in any direction tangential to the flux surface for nonsymmetric systems.

We find from Eqs. (36) and (40) that the radial neoclassical fluxes $\Gamma_{a j}^{\mathrm{bn}}(a=e, i, \cdots, j=0,1,2, \cdots)$ are written as linear combinations of the averaged flows $\left\langle B u_{\| a j}\right\rangle$ and the thermodynamic forces $\left(X_{a 1}, X_{a 2}, X_{E}\right)(a=e, i, \cdots)$. As mentioned 
after Eq. (33), when the Laguerre-polynomial expansion is truncated up to the order of $j=j_{\max }$, we can solve some appropriate energy-moment equations to express $\left\langle B u_{\| a j}\right\rangle$ in terms of $\left(X_{a 1}, X_{a 2}, X_{E}\right)$. Then, substituting these expressions for $\left\langle B u_{\| a j}\right\rangle$ into $\Gamma_{a j}^{\text {bn }}$, we can finally obtain the neoclassical transport equations which represent $\Gamma_{a j}^{\mathrm{bn}}$ by $\left(X_{a 1}, X_{a 2}, X_{E}\right)$. Here, it should be emphasized again that the expressions, which relate $\left\langle B u_{\| a j}\right\rangle$ to $\left(X_{a 1}, X_{a 2}, X_{E}\right)$, and accordingly the transport equations depend on what weight functions of $K$ are used in the energy integrals to derive the moment equations. This point will be further discussed in the following subsections.

\section{B. Sugama-Nishimura method}

In Sugama and Nishimura, ${ }^{14}$ the coefficients $\left[M_{a}(K), N_{a}(K), L_{a}(K)\right]$ are defined by

$$
\begin{aligned}
M_{a}(K)= & \frac{m_{a}^{2}}{T_{a}}\left[\nu_{D}^{a}(K)\right]^{2} D_{33}(K)\left[1-\frac{3 m_{a} \nu_{D}^{a}(K) D_{33}(K)}{2 T_{a} K\left\langle B^{2}\right\rangle}\right]^{-1}, \\
N_{a}(K)= & \frac{m_{a}}{T_{a}} \nu_{D}^{a}(K) D_{13}(K)\left[1-\frac{3 m_{a} \nu_{D}^{a}(K) D_{33}(K)}{2 T_{a} K\left\langle B^{2}\right\rangle}\right]^{-1}, \\
L_{a}(K)= & \frac{1}{T_{a}}\left(D_{11}(K)-\frac{B^{2} v^{2} \nu_{D}^{a}}{3 \Omega_{a}^{2}}\left\langle\widetilde{U}^{2}\right\rangle+\frac{3 m_{a} \nu_{D}^{a}(K)\left[D_{13}(K)\right]^{2}}{2 T_{a} K\left\langle B^{2}\right\rangle}\right. \\
& \left.\times\left[1-\frac{3 m_{a} \nu_{D}^{a}(K) D_{33}(K)}{2 T_{a} K\left\langle B^{2}\right\rangle}\right]^{-1}\right) .
\end{aligned}
$$

Then, by multiplication with $\left(m_{a} / T_{a}\right) \nu_{D}^{a}(K)[1$ $\left.-3 m_{a} \nu_{D}^{a}(K) D_{33}(K) /\left(2 T_{a} K\left\langle B^{2}\right\rangle\right)\right]^{-1}$, Eq. (33) is rewritten as

$$
\begin{aligned}
& M_{a}(K) \sum_{j=0}^{\infty} L_{j}^{(3 / 2)}(K)\left\langle B u_{\| a j}\right\rangle /\left\langle B^{2}\right\rangle+N_{a}(K)\left[X_{a 1}-X_{a 2} L_{j}^{(3 / 2)}(K)\right] \\
& =\frac{2}{3} \frac{K}{n_{a}}\left[\sum_{j=0}^{\infty} c_{j} L_{j}^{(3 / 2)}(K) \sum_{b} \sum_{k=0}^{\infty} l_{j+1, k+1}^{a b}\left\langle B u_{\| b k}\right\rangle\right. \\
& \left.\quad+n_{a} e_{a}\left\langle B^{2}\right\rangle^{1 / 2} X_{E}\right] .
\end{aligned}
$$

Next, using Eq. (42) to remove $X_{E}$ from Eq. (36), another expression for $\mathcal{F}_{a}^{\text {bn }}(K)$ is obtained with the aid of Eq. (41) as

$$
\begin{aligned}
\mathcal{F}_{a}^{\mathrm{bn}}(K)= & N_{a}(K) \sum_{j=0}^{\infty} L_{j}^{(3 / 2)}(K)\left\langle B u_{\| a j}\right\rangle /\left\langle B^{2}\right\rangle \\
& +L_{a}(K)\left[X_{a 1}-X_{a 2} L_{1}^{(3 / 2)}(K)\right] .
\end{aligned}
$$

Now, let us truncate the Laguerre-polynomial expansion up to the order of $j=j_{\max }$. Then, in the Sugama-Nishimura method, Eqs. (42) and (43) are multiplied with $n_{a}(2 / \sqrt{\pi}) \sqrt{K} e^{-K} L_{j}^{(3 / 2)}(K)\left(j=0,1, \cdots, j_{\max }\right)$ and they are integrated in $K$ to yield

$$
\begin{gathered}
\sum_{k=0}^{j_{\max }} M_{j+1, k+1}^{a}\left\langle B u_{\| a k}\right\rangle /\left\langle B^{2}\right\rangle+N_{j+1,1}^{a} X_{a 1}-N_{j+1,2}^{a} X_{a 2} \\
=\sum_{b} \sum_{k=0}^{j_{\max }} l_{j+1, k+1}^{a b}\left\langle B u_{\| b k}\right\rangle+\delta_{j 0} n_{a} e_{a}\left\langle B^{2}\right\rangle^{1 / 2} X_{E} \\
\left(j=0,1, \cdots, j_{\max }\right)
\end{gathered}
$$

and

$$
\begin{aligned}
\Gamma_{a j}^{\mathrm{bn}}= & \sum_{k=0}^{j_{\max }} N_{j+1, k+1}^{a}\left\langle B u_{\| a k}\right\rangle /\left\langle B^{2}\right\rangle+L_{j+1,1}^{a} X_{a 1}-L_{j+1,2}^{a} X_{a 2} \\
& \left(j=0,1, \cdots, j_{\max }\right),
\end{aligned}
$$

where

$$
\begin{aligned}
& {\left[M_{j+1, k+1}^{a}, N_{j+1, k+1}^{a}, L_{j+1, k+1}^{a}\right]} \\
& =n_{a} \frac{2}{\sqrt{\pi}} \int_{0}^{\infty} d K \sqrt{K} e^{-K} L_{j}^{(3 / 2)}(K) L_{k}^{(3 / 2)}(K) \\
& \quad \times\left[M_{a}(K), N_{a}(K), L_{a}(K)\right] .
\end{aligned}
$$

As seen from Ref. 14, the left-hand side of Eq. (44) equals the neoclassical parallel viscosities so that we can write

$$
\begin{aligned}
\Pi_{\| a j} & \equiv\left\langle\int d^{3} v f_{a 1} \sigma_{U a} L_{j}^{(3 / 2)}(K)\right\rangle \\
& =\sum_{k=0}^{j_{\max }} M_{j+1, k+1}^{a}\left\langle B u_{\| a k}\right\rangle /\left\langle B^{2}\right\rangle+N_{j+1,1}^{a} X_{a 1}-N_{j+1,2}^{a} X_{a 2}
\end{aligned}
$$

with $\sigma_{U a} \equiv-V\left(m_{a} v \xi B\right)$.

We find that $\Pi_{\| a j}, \Gamma_{a}^{\mathrm{bn}}=\Gamma_{a 0}^{\mathrm{bn}}$, and $q_{a}^{\mathrm{bn}} / T_{a}=-\Gamma_{a 1}^{\mathrm{bn}}$ are regarded as fluxes conjugate to driving forces represented by $\left\langle B u_{\| a j}\right\rangle /\left\langle B^{2}\right\rangle, X_{a 1}$, and $X_{a 2}$, respectively, ${ }^{14}$ and that these fluxes and forces are connected by the Onsager-symmetric coefficients as shown below from Eqs. (45) and (47). In order to show the Onsager symmetry clearly, Eqs. (45) and (47) are combined and rewritten in the matrix form as

$$
\left[\begin{array}{c}
\mathbf{P}_{\|} \\
\mathbf{G}^{\text {bn }}
\end{array}\right]=\left[\begin{array}{cc}
\mathbf{M} & \mathbf{N} \\
\mathbf{N}^{\text {tr }} & \mathbf{L}
\end{array}\right]\left[\begin{array}{l}
\mathbf{U}_{\|} \\
\mathbf{X}
\end{array}\right],
$$

where $\mathbf{M}, \mathbf{N}$, and $\mathbf{L}$ are $\left(j_{\max }+1\right) \times\left(j_{\max }+1\right),\left(j_{\max }+1\right) \times 2$, and $2 \times 2$ matrices which have components given by $M_{j+1, k+1}^{a}, \quad N_{j+1, k+1}^{a}, \quad$ and $L_{j+1, k+1}^{a}, \quad$ respectively, and $\mathbf{N}^{\mathrm{tr}}$ represents the transpose matrix of $\mathbf{N}$. The column vectors $\mathbf{P}_{\|}, \mathbf{G}^{\text {bn }}, \mathbf{U}_{\|}$, and $\mathbf{X}$ are defined by $\mathbf{P}_{\|}=\left[\Pi_{\| a 0}, \cdots, \Pi_{\| a j_{\max }}\right]^{\mathrm{tr}}$, $\mathbf{G}^{\mathrm{bn}}=\left[\Gamma_{a 0}^{\mathrm{bn}}, \Gamma_{a 1}^{\mathrm{bn}}\right]^{\mathrm{tr}}=\left[\Gamma_{a}^{\mathrm{bn}},-q_{a}^{\mathrm{bn}} / T_{a}\right]^{\mathrm{tr}}, \quad \mathbf{U}_{\|}=\left[\left\langle B u_{\| a 0}\right\rangle /\left\langle B^{2}\right\rangle, \cdots\right.$, $\left.\left\langle B u_{\| a j_{\max }}\right\rangle /\left\langle B^{2}\right\rangle\right]^{\text {tr }}$, and $\mathbf{X}=\left[X_{a 1},-X_{a 2}\right]^{\text {tr }}$, respectively. As seen above, $\quad\left[\Pi_{\| a 0}, \cdots, \Pi_{\| a j_{\max }}, \Gamma_{a}^{\mathrm{bn}},-q_{a}^{\mathrm{bn}} / T_{a}\right] \quad$ and $\quad\left[\left\langle B u_{\| a 0}\right\rangle\right.$ $\left./\left\langle B^{2}\right\rangle, \cdots,\left\langle B u_{\| a j_{\max }}\right\rangle /\left\langle B^{2}\right\rangle, X_{a 1},-X_{a 2}\right]$ are combined by a symmetric matrix and this symmetric relation is kept even if replacing $-q_{a}^{\mathrm{bn}} / T_{a}$ and $-X_{a 2}$ with $q_{a}^{\mathrm{bn}} / T_{a}$ and $X_{a 2}$, respectively. For the case of $j_{\max }=1$, the above matrix-form equation is equivalent to Eq. (35) in Ref. 14, which is written such that the signs of the even-numbered components of the column vectors are changed. 
Equations (44) and (45) are the basic equations in the Sugama-Nishimura method to determine the neoclassical transport coefficients. We note that Eqs. (44) and (45) are equivalent to a combination of Eqs. (35), (C1), and (C2) in Ref. 14 for the case of $j_{\max }=1$. Solving Eq. (44), the averaged flows $\left\langle B u_{\| a j}\right\rangle\left(j=0,1, \cdots, j_{\max }\right)$ are expressed by linear combinations of the thermodynamic forces $X_{a 1}, X_{a 2}$, and $X_{E}$. Then, substituting these expressions into Eq. (45) and

$$
J_{E} \equiv \sum_{a} n_{a} e_{a}\left\langle B u_{\| a}\right\rangle\left\langle\left\langle B^{2}\right\rangle^{1 / 2},\right.
$$

we finally obtain the neoclassical transport equations which relate the radial transport fluxes $\Gamma_{a j}^{\mathrm{bn}}$ and the parallel electric current $J_{E}$ to the thermodynamic forces $X_{a 1}, X_{a 2}$, and $X_{E}$. Especially, the transport equations derived from the SugamaNishimura method show that $\Gamma_{a}^{\mathrm{bn}} \equiv \Gamma_{a 0}^{\mathrm{bn}}, q_{a}^{\mathrm{bn}} / T_{a} \equiv-\Gamma_{a 1}^{\mathrm{bn}}$, and $J_{E}$ are connected by their conjugate forces $X_{a 1}, X_{a 2}$, and $X_{E}$ by the Onsager-symmetric transport coefficients.

It should be noted that the right-hand side of Eq. (44) represents the sum of the averaged parallel electric force (appearing only for the $j=0$ case) and the averaged parallel friction forces written by

$$
\left\langle B \int d^{3} v C_{a}\left(f_{a 1}\right) m_{a} v_{\|} L_{j}^{(3 / 2)}(K)\right\rangle .
$$

Thus, for the $j=0$ case, Eq. (44) represents the momentum balance equation,

$$
\Pi_{\| a 0} \equiv\left\langle\mathbf{B} \cdot\left(\nabla \cdot \boldsymbol{\pi}_{a}\right)\right\rangle=\left\langle B F_{\| a 1}\right\rangle+n_{a} e_{a}\left\langle B E_{\|}\right\rangle,
$$

where $\quad \boldsymbol{\pi}_{a} \equiv \int d^{3} v m_{a}\left(v_{\|}^{2}-\frac{1}{2} v_{\perp}^{2}\right) f_{a 1}\left(\mathbf{b b}-\frac{1}{3} \mathbf{I}\right) \quad$ and $\quad F_{\| a 1}$ $\equiv \int d^{3} v C_{a}\left(f_{a 1}\right) m_{a} v_{\|}=\Sigma_{b} \Sigma_{k=0}^{j_{\max }} l_{1, k+1}^{a b} u_{\| b k}$ denote the viscosity tensor and the parallel friction force, respectively. Then, using the momentum-conservation property given by Eq. (26), the charge neutrality condition $\Sigma_{a} n_{a} e_{a}=0$, and taking the species summation of Eq. (50) yields

$$
\sum_{a}\left\langle\mathbf{B} \cdot\left(\nabla \cdot \boldsymbol{\pi}_{a}\right)\right\rangle=0 .
$$

As shown in Appendix D of Ref. 14, for symmetric cases, where $c_{1} \partial B / \partial \theta+c_{2} \partial B / \partial \zeta=0$ holds $\left(c_{1}=0, c_{2}=0\right.$, and $c_{1} \cdot c_{2}$ $\neq 0$ represent the axisymmetric, poloidally symmetric, and helically symmetric cases, respectively), we have $N_{j+1, k+1}^{a} / M_{j+1, k+1}^{a}=L_{j+1, k+1}^{a} / N_{j+1, k+1}^{a}=N_{a}(K) / M_{a}(K)=L_{a}(K)$ $/ N_{a}(K)$ and therefore obtain $\Gamma_{a j}^{\mathrm{bn}} \propto \prod_{\| a j}$ from Eqs. (45) and (47). Finally, combining this fact for the $j=0$ case, $\Gamma_{a}^{\mathrm{bn}}$ $\propto\left\langle\mathbf{B} \cdot\left(\nabla \cdot \boldsymbol{\pi}_{a}\right)\right\rangle$, with Eq. (51), we see that, when applied to the symmetric cases, the Sugama-Nishimura method guarantees the intrinsic ambipolarity condition,

$$
\sum_{a} \Gamma_{a}^{\mathrm{bn}}=0 \quad \text { (for symmetric systems). }
$$

\section{Taguchi's method}

In Taguchi's method, ${ }^{15}$ we multiply Eq. (33) with $n_{a}(2 / \sqrt{\pi}) \sqrt{K} e^{-K} L_{j}^{(3 / 2)}(K)\left(j=0,1, \cdots, j_{\max }\right)$ and take the $K$ integrals to obtain

$$
\begin{gathered}
\sum_{k=0}^{j_{\max }} A_{j+1, k+1}^{a}\left\langle B u_{\| a k}\right\rangle /\left\langle B^{2}\right\rangle+B_{j+1,1}^{a} X_{a 1}-B_{j+1,2}^{a} X_{a 2} \\
=\frac{1}{n_{a}} \sum_{m=0}^{j_{\max }} Z_{j+1, m+1}^{a} c_{m} \sum_{b} \sum_{k=0}^{j_{\max }} l_{m+1, k+1}^{a b}\left\langle B u_{\| b k}\right\rangle /\left\langle B^{2}\right\rangle \\
\quad+Z_{j+1,1}^{a} e_{a} X_{E} /\left\langle B^{2}\right\rangle^{1 / 2} \quad\left(j=0,1, \cdots, j_{\max }\right),
\end{gathered}
$$

where the Laguerre-polynomial expansion is truncated up to the order of $j=j_{\max }$. Here, the coefficients $A_{j+1, k+1}^{a}, B_{j+1, k+1}^{a}$, and $Z_{j+1, k+1}^{a}$ are defined by

$$
\begin{aligned}
{\left[\begin{array}{c}
A_{j+1, k+1}^{a} \\
B_{j+1, k+1}^{a} \\
Z_{j+1, k+1}^{a}
\end{array}\right]=} & n_{a} \frac{2}{\sqrt{\pi}} \int_{0}^{\infty} d K \sqrt{K} e^{-K} L_{j}^{(3 / 2)}(K) L_{k}^{(3 / 2)}(K) \\
& \times\left[\begin{array}{c}
m_{a} \nu_{D}^{a}(K) D_{33}(K) \\
D_{13}(K) \\
-D_{33}(K)+2 T_{a} K\left\langle B^{2}\right\rangle / 3 m_{a} \nu_{D}^{a}(K)
\end{array}\right] .
\end{aligned}
$$

It should be noted that, in the Sugama-Nishimura method, the additional factor $\left(m_{a} / T_{a}\right) \nu_{D}^{a}(K)\left[1-3 m_{a} \nu_{D}^{a}(K) D_{33}(K)\right.$ $\left./\left(2 T_{a} K\left\langle B^{2}\right\rangle\right)\right]^{-1}$ is multiplied for taking the energy integral to derive Eq. (44) so that it coincides with the same parallel momentum equations as used in the conventional moment approach for calculation of the neoclassical transport coefficients. ${ }^{28-31}$ Next, we multiply Eq. (36) with $n_{a}(2 / \sqrt{\pi}) \sqrt{K} e^{-K} L_{j}^{(3 / 2)}(K)\left(j=0,1, \cdots, j_{\max }\right)$ and take the energy integrals to get the radial neoclassical fluxes [see Eq. (40)],

$$
\begin{aligned}
\Gamma_{a j}^{\mathrm{bn}}= & \sum_{k=0}^{j_{\max }} \mathcal{N}_{j+1, k+1}^{a}\left\langle B u_{\| a k}\right\rangle /\left\langle B^{2}\right\rangle+\frac{1}{n_{a} T_{a}} \sum_{m=0}^{j_{\max }} B_{j+1, m+1}^{a} \\
& \times c_{m} \sum_{b} \sum_{k=0}^{j_{\max }} l_{m+1, k+1}^{a b}\left\langle B u_{\| b k}\right\rangle /\left\langle B^{2}\right\rangle+\mathcal{L}_{j+1,1}^{a} X_{a 1} \\
& -\mathcal{L}_{j+1,2}^{a} X_{a 2} \quad\left(j=0,1, \cdots, j_{\max }\right),
\end{aligned}
$$

where the coefficients $\mathcal{N}_{j+1, k+1}^{a}$ and $\mathcal{L}_{j+1, k+1}^{a}$ are defined by

$$
\begin{aligned}
{\left[\begin{array}{c}
\mathcal{N}_{j+1, k+1}^{a} \\
\mathcal{L}_{j+1, k+1}^{a}
\end{array}\right]=} & n_{a} \frac{2}{\sqrt{\pi}} \int_{0}^{\infty} d K \sqrt{K} e^{-K} L_{j}^{(3 / 2)}(K) L_{k}^{(3 / 2)}(K) \\
& \times\left[\begin{array}{c}
\left(m_{a} / T_{a}\right) \nu_{D}^{a}(K) D_{13}(K) \\
\left(1 / T_{a}\right)\left[D_{11}(K)-\left(B^{2} v^{2} \nu_{D}^{a} / 3 \Omega_{a}^{2}\right)\left\langle\widetilde{U}^{2}\right\rangle\right]
\end{array}\right] .
\end{aligned}
$$

Recall that Eq. (45) is used in the Sugama-Nishimura method instead of Eq. (55) in order to retain the Onsagersymmetric relations of $\left[\Pi_{\| a j}, \Gamma_{a}^{\mathrm{bn}} \equiv \Gamma_{a 0}^{\mathrm{bn}}, q_{a}^{\mathrm{bn}} / T_{a}=-\Gamma_{a 1}^{\mathrm{bn}}\right]$ to $\left[\left\langle B u_{\| a j}\right\rangle /\left\langle B^{2}\right\rangle, X_{a 1}, X_{a 2}\right]$ as shown in Eqs. (45) and (47).

Equations (53) and (55) form the basic equations in Taguchi's method, from which the neoclassical transport coefficients are determined. Note that Eqs. (53) and (55) are equivalent to Eqs. (22) and (23) and Eqs. (40)-(46) in Ref. 15 , respectively, although our notations used here are different from those by Taguchi. ${ }^{15}$ Solving Eq. (53) gives expressions of the averaged flows $\left\langle B u_{\| a j}\right\rangle\left(j=0,1, \cdots, j_{\max }\right)$ in terms of linear combinations of the thermodynamic forces $X_{a 1}, X_{a 2}$, 
and $X_{E}$. Substituting them into Eqs. (55) and (48) yields the neoclassical transport coefficients, which relate the radial transport fluxes $\Gamma_{a j}^{\mathrm{bn}}$ and the parallel electric current $J_{E}$ to the thermodynamic forces $X_{a 1}, X_{a 2}$, and $X_{E}$.

In principle, the Sugama-Nishimura method and Taguchi's should lead to the same results in the limit of $j_{\max }$ $\rightarrow \infty$. However, different results are given from these methods for the finite value of $j_{\max }$. We see that Taguchi's method does not assure the Onsager symmetry of the neoclassical transport coefficients. Furthermore, Taguchi's method described in Secs. III and IV of Ref. 15 for nonaxisymmetric systems, which are equivalent to Eqs. (53) and (55) here, does not exactly reproduce the intrinsic ambipolar condition, Eq. (52), in the axisymmetric limit. It is noted that, in Sec. V of Ref. 15, Taguchi gives separately the method for the axisymmetric limit, which satisfies the ambipolar condition. In the next section, numerical examples are presented to illustrate these differences between results from the SugamaNishimura and Taguchi methods.

\section{NUMERICAL EXAMPLES}

In this section, in order to elucidate the differences between results from the Sugama-Nishimura and Taguchi methods, we consider the axisymmetric case, in which the magnetic field is given by

$$
\mathbf{B}=I(s) \nabla \zeta+\chi^{\prime}(s) \nabla \zeta \times \nabla s .
$$

Also, for simplicity, numerical examples only for the neoclassical transport of a single species of ions with charge $e$ in the banana regime are shown and small $\left(m_{e} / m_{i}\right)^{1 / 2}$-order effects associated with the electron transport are neglected. Then, we neglect effects of the parallel electric field and of ion-electron collisions on the drift kinetic equation for ions.

In the present case, the basic equations of the SugamaNishimura method in Eqs. (44) and (45) reduce to

$$
\begin{aligned}
& \sum_{k=0}^{j_{\max }}\left[M_{j+1, k+1} /\left\langle B^{2}\right\rangle-l_{j+1, k+1}^{i i}\right]\left\langle B u_{\| k}\right\rangle \\
& \quad=-N_{j+1,1} X_{1}+N_{j+1,2} X_{2} \quad\left(j=0,1, \cdots, j_{\max }\right),
\end{aligned}
$$

and

$$
\begin{aligned}
\Gamma_{j}^{\mathrm{b}}= & \sum_{k=0}^{j_{\max }} N_{j+1, k+1}\left\langle B u_{\| k}\right\rangle /\left\langle B^{2}\right\rangle+L_{j+1,1} X_{1}-L_{j+1,2} X_{2} \\
& \left(j=0,1, \cdots, j_{\max }\right),
\end{aligned}
$$

respectively, where the parallel flows $u_{\| j}$, the neoclassical banana fluxes $\Gamma_{j}^{\mathrm{b}}$, the thermodynamic forces $\left(X_{1}, X_{2}\right)$, and the matrix coefficients $\left(M_{j+1, k+1}, N_{j+1, k+1}, L_{j+1, k+1}\right)$ are all for ions, although the ion-species subscript $i$ is omitted. Values of the ion-ion collisional friction coefficients $l_{j+1, k+1}^{i i}$ are shown in the Appendix. The momentum conservation in ionion collisions is represented by $l_{1, k+1}^{i i}=l_{k+1,1}^{i i}=0(k$ $=0,1,2, \cdots)$.
On the other hand, the basic equations of Taguchi's method in Eqs. (53) and (55) are written here as

$$
\begin{gathered}
\sum_{k=0}^{j_{\max }}\left[A_{j+1, k+1}-\frac{1}{n_{i}} \sum_{m=0}^{j_{\max }} Z_{j+1, m+1} c_{m} l_{m+1, k+1}^{i i}\right] \frac{\left\langle B u_{\| k}\right\rangle}{\left\langle B^{2}\right\rangle} \\
\quad=-B_{j+1,1} X_{1}+B_{j+1,2} X_{2} \quad\left(j=0,1, \cdots, j_{\max }\right)
\end{gathered}
$$

and

$$
\begin{aligned}
\Gamma_{j}^{\mathrm{b}}= & \sum_{k=0}^{j_{\max }}\left[\mathcal{N}_{j+1, k+1}+\frac{1}{n_{i} T_{i}} \sum_{m=0}^{j_{\max }} B_{j+1, m+1} c_{m} l_{m+1, k+1}^{i i}\right] \\
& \times \frac{\left\langle B u_{\| k}\right\rangle}{\left\langle B^{2}\right\rangle}+\mathcal{L}_{j+1,1} X_{a 1}-\mathcal{L}_{j+1,2} X_{a 2} \\
& \left(j=0,1, \cdots, j_{\max }\right) .
\end{aligned}
$$

When all ions are assumed to lie in the banana regime for the axisymmetric case, we can analytically express the coefficients $D_{j k}(K)(j, k=1,3)$ in Eq. $(16)$ as $^{14}$

$$
\begin{aligned}
& D_{11}(K)=\frac{2 c^{2} m_{i} T_{i}}{3 e^{2}} K \nu_{D}^{i}(K)\left[f_{t} \frac{I^{2}}{\left(\chi^{\prime}\right)^{2}\left\langle B^{2}\right\rangle}+\left\langle\widetilde{U}^{2}\right\rangle\right], \\
& D_{13}(K)=-f_{t} \frac{2 c T_{i} I}{3 e \chi^{\prime}} K, \\
& D_{33}(K)=f_{t}\left\langle B^{2}\right\rangle \frac{2 T_{i}}{3 m_{i}} \frac{K}{\nu_{D}^{i}(K)} .
\end{aligned}
$$

Here, the fraction $f_{t}$ of trapped particles is defined by

$$
\begin{aligned}
f_{t} & \equiv 1-f_{c}, \\
f_{c} & \equiv \frac{3}{4}\left\langle B^{2}\right\rangle \int_{0}^{1 / B_{\max }} \frac{\lambda d \lambda}{\left.\left\langle(1-\lambda B)^{1 / 2}\right)\right\rangle},
\end{aligned}
$$

where $B_{\max }$ is the maximum value of the field strength over the flux surface. Also, $\widetilde{U}$ and $\left\langle\widetilde{U}^{2}\right\rangle$ are explicitly written for the axisymmetric case as

$$
\begin{aligned}
& \tilde{U}=\frac{I}{\chi^{\prime}}\left(\frac{1}{B}-\frac{B}{\left\langle B^{2}\right\rangle}\right), \\
& \left\langle\tilde{U}^{2}\right\rangle=\frac{I^{2}}{\left(\chi^{\prime}\right)^{2}}\left(\left\langle\frac{1}{B^{2}}\right\rangle-\frac{1}{\left\langle B^{2}\right\rangle}\right) .
\end{aligned}
$$

Now, substituting Eq. (62) into Eq. (41) yields

$$
\begin{aligned}
& M(K)=\frac{2}{3} \frac{f_{t}}{f_{c}} m_{i}\left\langle B^{2}\right\rangle K \nu_{D}^{i}(K), \\
& \frac{N(K)}{M(K)}=\frac{L(K)}{N(K)}=-\frac{c I}{e \chi^{\prime}\left\langle B^{2}\right\rangle} .
\end{aligned}
$$




$$
\begin{aligned}
& M_{j+1, k+1}=\frac{f_{t}}{f_{c}} \frac{n_{i} m_{i}}{\tau_{i i}}\left\langle B^{2}\right\rangle \hat{\mu}_{j+1, k+1}, \\
& \frac{N_{j+1, k+1}}{M_{j+1, k+1}}=\frac{L_{j+1, k+1}}{N_{j+1, k+1}}=-\frac{c I}{e \chi^{\prime}\left\langle B^{2}\right\rangle},
\end{aligned}
$$

where the dimensionless viscosity coefficients $\hat{\mu}_{j+1, k+1}$ are defined by

$$
\hat{\mu}_{j+1, k+1} \equiv\left\{\tau_{i i} \nu_{D}^{i}(K) L_{j}^{(3 / 2)}(K) L_{k}^{(3 / 2)}(K)\right\} .
$$

Substituting Eq. (62) into Eqs. (54) and (56) gives

$$
\begin{aligned}
& A_{j+1, k+1}=f_{t} n_{i} T_{i}\left\langle B^{2}\right\rangle \frac{\delta_{j k}}{c_{j}}, \\
& \mathcal{N}_{j+1, k+1}=-f_{t} \frac{c I}{e \chi^{\prime}} \frac{n_{i} m_{i}}{\tau_{i i}} \hat{\mu}_{j k}, \\
& Z_{j+1, k+1}=f_{c} \frac{n_{i} T_{i} \tau_{i i}}{m_{i}}\left\langle B^{2}\right\rangle \frac{\hat{\eta}_{j k}}{c_{j} c_{k}}, \\
& \frac{B_{j+1, k+1}}{A_{j+1, k+1}}=\frac{\mathcal{L}_{j+1, k+1}}{\mathcal{N}_{j+1, k+1}}=-\frac{c I}{e \chi^{\prime}\left\langle B^{2}\right\rangle},
\end{aligned}
$$

where the dimensionless coefficients $\hat{\eta}_{j+1, k+1}$ are defined by

$$
\hat{\eta}_{j+1, k+1} \equiv c_{j} c_{k}\left\{\frac{L_{j}^{(3 / 2)}(K) L_{k}^{(3 / 2)}(K)}{\tau_{i i} \nu_{D}^{i}(K)}\right\} .
$$

Here, we also define the dimensionless ion-ion friction coefficients $\hat{l}_{j+1, k+1}$ by

$$
\hat{l}_{j+1, k+1} \equiv-\frac{\tau_{i i}}{n_{i} m_{i}} l_{j+1, k+1}^{i i}
$$

Numerical values of the dimensionless coefficients $\hat{\mu}_{j+1, k+1}$, $\hat{\eta}_{j+1, k+1}$, and $\hat{l}_{j+1, k+1}$ are presented in the Appendix.

For axisymmetric systems, we can write the parallel flows $\left\langle B u_{\| j}\right\rangle(j=0,1,2, \cdots)$ in the forms of

$$
\begin{aligned}
& \left\langle B u_{\|}\right\rangle=\left\langle B u_{\| 0}\right\rangle=\left\langle B^{2}\right\rangle u_{\theta}+\frac{c I}{e \chi^{\prime}} X_{1}, \\
& \left\langle B q_{\|}\right\rangle=-\frac{5}{2} p_{i}\left\langle B u_{\| 1}\right\rangle=\left\langle B^{2}\right\rangle q_{\theta}+\frac{5}{2} p_{i} \frac{c I}{e \chi^{\prime}} X_{2}, \\
& \left\langle B u_{\| j}\right\rangle=\left\langle B^{2}\right\rangle u_{j \theta} \quad(j \geqslant 2),
\end{aligned}
$$

where $u_{\theta}$ is expressed in terms of the poloidal component of the flow vector $\mathbf{u}$ as $u_{\theta}=\left(V^{\prime} / 4 \pi^{2} \chi^{\prime}\right)\langle\mathbf{u} \cdot \nabla \theta\rangle$ and similar expressions are given for $q_{\theta}$ and $u_{j \theta}(j \geqslant 2)$.

Now, based on the Sugama-Nishimura method, we use Eqs. (58) and (59), into which Eqs. (66) and (71) are substituted, to express the poloidal flows (instead of the parallel flows) and the radial transport fluxes in terms of the thermodynamic forces. First, it should be noted that, for any value of $j_{\text {max }}$, the radial ion particle flux vanishes,

$$
\Gamma^{\mathrm{b}} \equiv \Gamma_{0}^{\mathrm{b}}=0
$$

which corresponds to the intrinsic ambipolar condition in Eq. (52) for the case of the small- $\left(m_{e} / m_{i}\right)^{1 / 2}$ limit. Also, the radial heat flux $q^{\mathrm{b}} \equiv-T_{i} \Gamma_{1}^{\mathrm{b}}$ determined from Eqs. (58) and (59) is independent of $X_{1}$, which implies the Onsager symmetry. Recall that the radial fluxes $\Gamma_{0}^{\mathrm{b}}$ and $\Gamma_{1}^{\mathrm{b}}$ are conjugate to the forces $X_{1}$ and $X_{2}$, respectively, so that, from the Onsager symmetry, the coefficient connecting $\Gamma_{1}^{\mathrm{b}}$ with $X_{1}$ should vanish when the coefficient connecting $\Gamma_{0}^{\mathrm{b}}$ with $X_{2}$ vanishes. The results are written as

$$
\begin{aligned}
& {\left[\begin{array}{c}
u_{\theta} \\
\frac{2}{5 p_{i}} q_{\theta}
\end{array}\right]=-\frac{c I X_{2}}{e \chi^{\prime}\left\langle B^{2}\right\rangle}\left[\begin{array}{c}
C_{0 \theta} \\
C_{1 \theta}
\end{array}\right],} \\
& q^{\mathrm{b}} \equiv-T_{i} \Gamma_{1}^{\mathrm{b}}=C_{q} \frac{f_{t}}{f_{c}} \frac{n_{i} m_{i} T_{i} c^{2} I^{2}}{\left.\chi^{\prime}\right)^{2}\left\langle B^{2}\right\rangle \tau_{i i}} X_{2},
\end{aligned}
$$

where numerical values of the dimensionless coefficients $C_{0 \theta}, C_{1 \theta}$, and $C_{q}$ obtained from the Sugama-Nishimura method are shown by solid curves in Fig. 1 for the cases of $j_{\text {max }}=1,2$, and 3 (referred to as the $13 \mathrm{M}, 21 \mathrm{M}$, and $29 \mathrm{M}$ approximations, respectively, in Ref. 29). Especially, for the case of $j_{\max }=1$, we obtain

$$
\begin{aligned}
{\left[\begin{array}{c}
C_{0 \theta} \\
C_{1 \theta} \\
C_{q}
\end{array}\right]=} & \frac{\hat{l}_{22}}{\hat{l}_{22} \hat{\mu}_{11}+\left(f_{t} / f_{c}\right)\left[\hat{\mu}_{11} \hat{\mu}_{22}-\left(\hat{\mu}_{12}\right)^{2}\right]} \\
& \times\left[\begin{array}{c}
\hat{\mu}_{12} \\
\hat{\mu}_{11} \\
\hat{\mu}_{11} \hat{\mu}_{22}-\left(\hat{\mu}_{12}\right)^{2}
\end{array}\right] \\
\simeq & \frac{1}{1+0.461474\left(f_{t} / f_{c}\right)}\left[\begin{array}{c}
1.17295 \\
1 \\
0.652622
\end{array}\right] .
\end{aligned}
$$

Here, we should repeat that the Sugama-Nishimura method and the results shown in Eqs. (73) and (74) for the ion banana transport in the axisymmetric system are equivalent to those of the conventional moment approach. ${ }^{28-30}$

On the other hand, Taguchi's method leads us to substitute Eq. (68) into Eqs. (60) and (61), from which the ion poloidal flows and radial fluxes are derived as

$$
\begin{aligned}
& {\left[\begin{array}{c}
u_{\theta} \\
\frac{2}{5 p_{i}} q_{\theta}
\end{array}\right]=-\frac{c I X_{2}}{e \chi^{\prime}\left\langle B^{2}\right\rangle}\left[\begin{array}{c}
C_{0 \theta}^{\prime} \\
C_{1 \theta}^{\prime}
\end{array}\right],} \\
& {\left[\begin{array}{c}
\Gamma^{\mathrm{b}} \\
q^{\mathrm{b}}
\end{array}\right] \equiv\left[\begin{array}{c}
\Gamma_{0}^{\mathrm{b}} \\
-T_{i} \Gamma_{1}^{\mathrm{b}}
\end{array}\right]=\frac{f_{t}}{f_{c}} \frac{n_{i} m_{i} T_{i} c^{2} I^{2}}{\left.\chi^{\prime}\right)^{2}\left\langle B^{2}\right\rangle \tau_{i i}} X_{2}\left[\begin{array}{c}
C_{\Gamma}^{\prime} \\
C_{q}^{\prime}
\end{array}\right],}
\end{aligned}
$$

where numerical values of the dimensionless coefficients $C_{0 \theta}^{\prime}, C_{1 \theta}^{\prime}, C_{\Gamma}^{\prime}$, and $C_{q}^{\prime}$ are shown by dotted curves in Figs. 1 and 2 for the cases of $j_{\max }=1,2$, and 3 . For the case of $j_{\text {max }}=1$, we have 
(a)

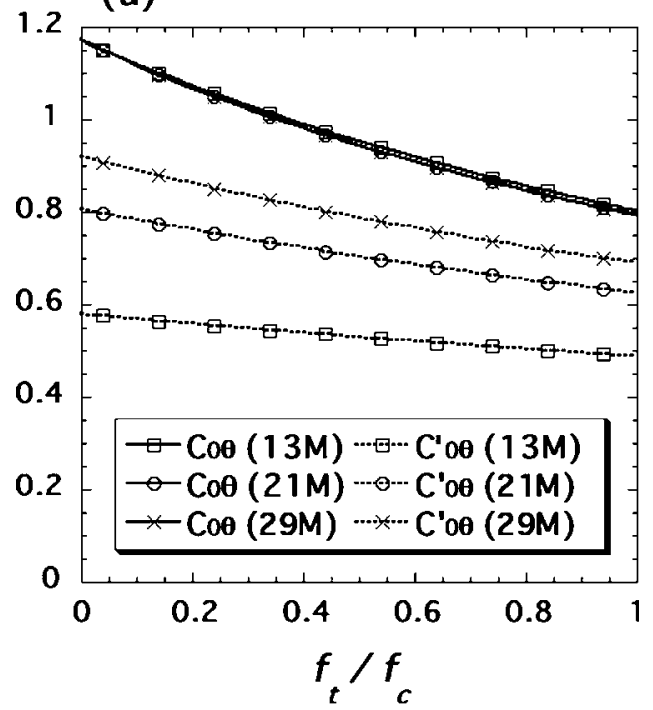

(b)

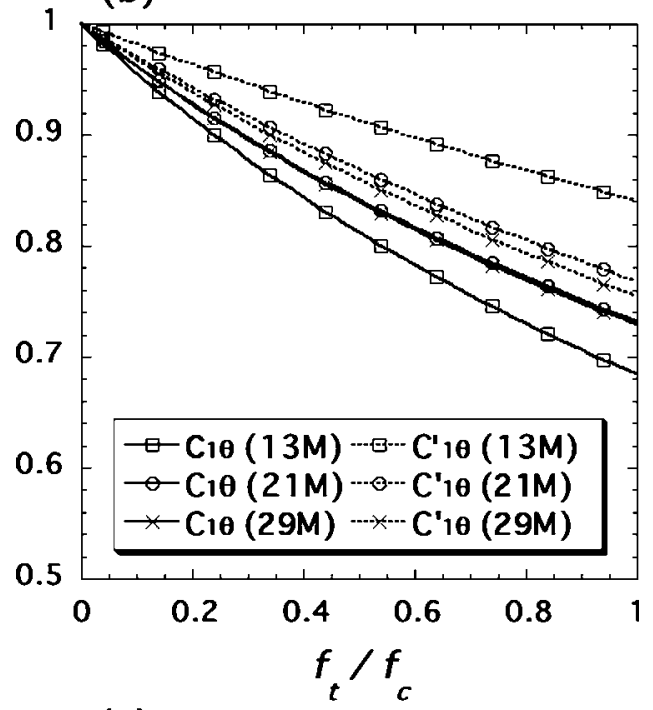

(c)

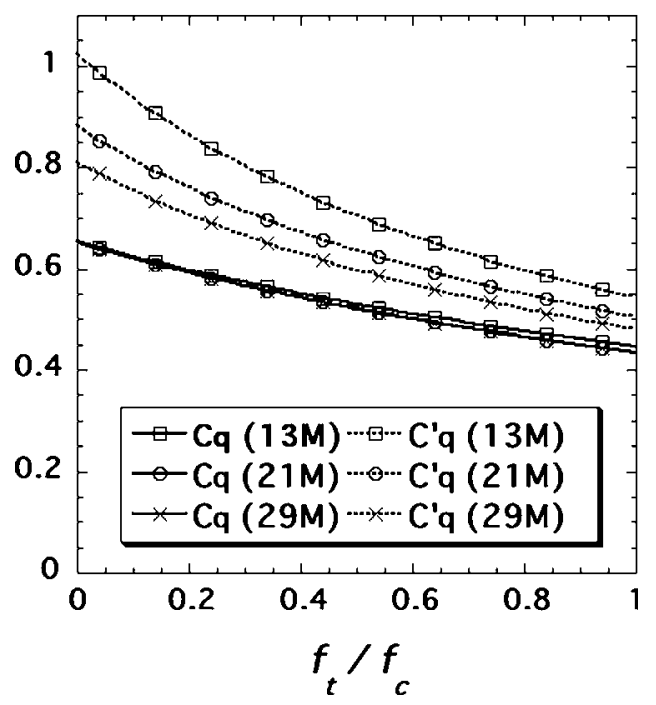

FIG. 1. Dimensionless neoclassical coefficients calculated as functions of $f_{t} / f_{c}$ for $j_{\max }=1(13 \mathrm{M}), 2(21 \mathrm{M})$, and $3(29 \mathrm{M})$. The coefficients $C_{0 \theta}, C_{1 \theta}$, and $C_{q}$ in Eq. (73) obtained from the Sugama-Nishimura method are plotted by solid curves in (a), (b), and (c), respectively. For comparison, also plotted by dotted curves are $C_{0 \theta}^{\prime}, C_{1 \theta}^{\prime}$, and $C_{q}^{\prime}$ in Eq. (75) obtained from Taguchi's method.

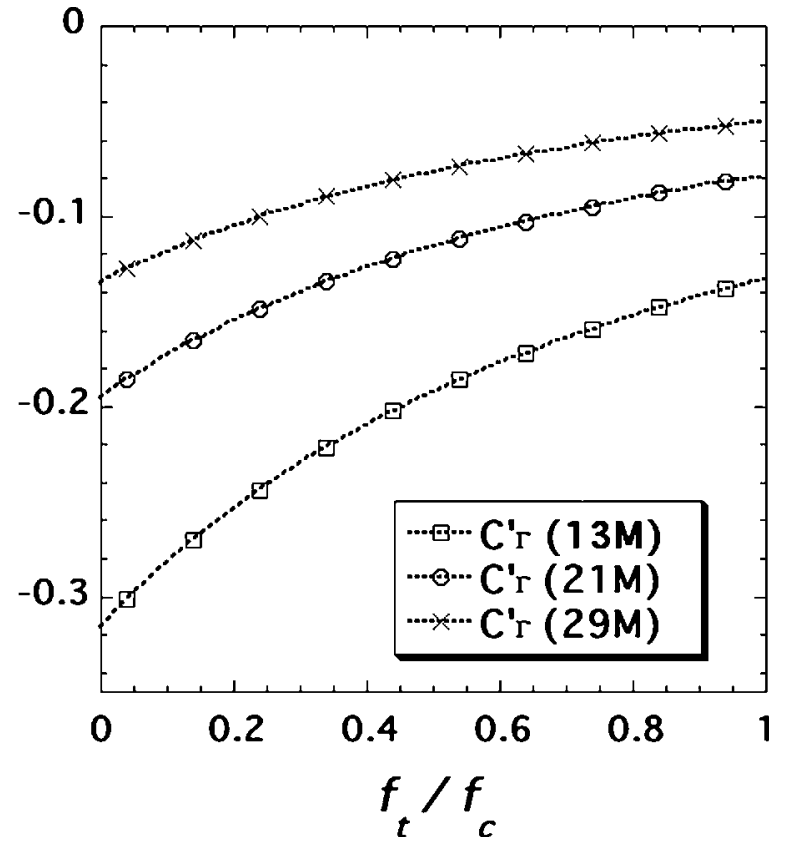

FIG. 2. The dimensionless coefficient $C_{\Gamma}^{\prime}$ for the fictitious radial particle flux in Eq. (75) as a function of $f_{t} / f_{c}$ obtained from Taguchi's method for $j_{\max }=1(13 \mathrm{M}), 2(21 \mathrm{M})$, and $3(29 \mathrm{M})$.

$$
\begin{aligned}
{\left[\begin{array}{c}
C_{0 \theta}^{\prime} \\
C_{1 \theta}^{\prime} \\
C_{\Gamma}^{\prime} \\
C_{q}^{\prime}
\end{array}\right] } & =\frac{\hat{l}_{22}}{\hat{l}_{22} \hat{\eta}_{22}+\left(f_{t} / f_{c}\right)} \times\left[\begin{array}{c}
-\hat{\eta}_{12} \\
\hat{\eta}_{22} \\
-f_{c}\left(\hat{\mu}_{11} \hat{\eta}_{12}+\hat{\mu}_{12} \hat{\eta}_{22}\right) \\
f_{c}\left(\hat{\mu}_{12} \hat{\eta}_{12}+\hat{\mu}_{22} \hat{\eta}_{22}\right)+f_{t}
\end{array}\right] \\
& \simeq \frac{1}{1+2.62916\left(f_{t} / f_{c}\right)}\left[\begin{array}{c}
0.580094 \\
1 \\
-0.315896 f_{c} \\
1.02315-0.754204 f_{t}
\end{array}\right] .
\end{aligned}
$$

Here, the nonzero value of the coefficient $C_{\Gamma}^{\prime}$ for the fictitious radial particle flux $\Gamma^{\mathrm{b}}$ indicates that the ambipolar condition is violated and that the Onsager symmetry is broken because the reciprocal coefficient for $C_{\Gamma}^{\prime}$, which connects $q^{\mathrm{b}}$ with $X_{1}$, still vanishes as seen in Eq. (75). It should be recalled here that the above results are derived from the axisymmetric limit of Taguchi's formulas for general toroidal systems (but not from his formulas given separately for the axisymmetric (ase). ${ }^{15}$

It is claimed in Sec. II that results from the SugamaNishimura and Taguchi methods should be equivalent to each other in the limit of $j_{\max } \rightarrow \infty$. This fact is directly proven for the present case by noting that

$$
\sum_{m=0}^{\infty} \hat{\mu}_{j m} \hat{\eta}_{m k}=\delta_{j k} \quad(j, k=0,1,2, \cdots) .
$$

However, as seen from Fig. 1, the coefficients $C_{0 \theta}, C_{1 \theta}$, and $C_{q}$ obtained from the Sugama-Nishimura method (or the conventional moment approach) give better convergence with increasing $j_{\max }$ than the corresponding coefficients $C_{0 \theta}^{\prime}$, $C_{1 \theta}^{\prime}$, and $C_{q}^{\prime}$ from Taguchi's. Especially, the moment ap- 
proach shows the convergent results even for $j_{\max }=1$ in the limit of small $f_{t}$ (or the large aspect ratio), in which Eq. (73) gives the radial neoclassical ion heat flux as

$$
\left\langle\mathbf{q}_{i} \cdot \nabla r\right\rangle \simeq 0.477 \frac{n_{i} \rho_{T i}^{2} q^{2}}{\epsilon^{3 / 2} \tau_{i i}} \frac{\partial T_{i}}{\partial r}
$$

Here, $\rho_{T i} \equiv c \sqrt{2 m_{i} T_{i}} /\left(e B_{0}\right)$ represents the ion thermal gyroradius, $q$ is the safety factor, and $\epsilon \equiv r / R_{0}(\ll 1)$ is the inverse aspect ratio for the toroid with the concentric circular cross section of the minor radius $r$, where the fraction $f_{t}$ of trapped particles is given by Eq. (A4) in the Appendix. We also note that the ion-ion collision time $\tau_{i i}$ used here is related to the ion collision time $\tau_{i}$ of Braginskii ${ }^{32}$ by $\tau_{i}=\sqrt{2} \tau_{i i}$. The result in Eq. (78) agrees with the one derived by Rosenbluth et al. ${ }^{33}$ using the variational principle, in which the numerical coefficient is given as 0.48. On the other hand, Eq. (75) shows that the value of the coefficient is given as 0.748 from Taguchi's method for $j_{\max }=1$.

\section{CONCLUSIONS}

In this work, a detailed comparison is made between two moment-equation methods for calculating neoclassical transport coefficients, which are proposed by Sugama and Nishimura and by Taguchi. Both methods are derived from the drift kinetic equation with the same collision model in order to correctly include the effects of collisional momentum transfer and they both use the Laguerre-polynomial expansion to represent the guiding-center distribution function associated with the parallel flows of particles, heat, and other higher-order energy moments. Also, these methods are given here in the forms applicable for an arbitrary truncation number of the Laguerre-polynomial expansion, which are useful for improving the accuracies. The two methods are equivalent with each other in the limit that the truncation number goes to infinity. However, a difference appears between their results when the expansion is truncated up to a finite order because different weight functions are used in them to derive the moment equations that relate the finite number of the parallel flows to the thermodynamic forces.

In the Sugama-Nishimura method, the relations between the parallel flows and the thermodynamic forces are determined by the same momentum balance equations as used in the conventional moment approach so that the intrinsic ambipolarity of particle fluxes is exactly derived in the symmetric systems at each order of the truncation. Furthermore, the resultant neoclassical transport coefficients from their method satisfy the Onsager symmetry. On the other hand, the above properties are not retained exactly in the results from Taguchi's method. The differences between results from the two methods are demonstrated by numerical examples for the ion banana neoclassical transport in the axisymmetric case, where a better convergence of the transport coefficients is also confirmed for a smaller truncation number of the Laguerre-polynomial expansion in the Sugama-Nishimura method. Thus, their method is considered to be appropriate especially for investigating the neoclassical transport in quasisymmetric systems and in tokamaks with the axisymmetry partially broken, where it is important to accurately evaluate how the transport differs from the exactly-symmetric limit.

\section{ACKNOWLEDGMENTS}

The authors acknowledge stimulating discussion with Dr. D. A. Spong, Dr. C. D. Beidler, and Dr. M. Yokoyama. This work is supported in part by the Japanese Ministry of Education, Culture, Sports, Science, and Technology, Grant No. 16560727, and in part by the NIFS Collaborative Research Program, NIFS06KDAD006.

\section{APPENDIX: DIMENSIONLESS COEFFICIENTS IN SEC. III}

Numerical values of the dimensionless coefficients $\hat{\mu}_{j+1, k+1}, \hat{\eta}_{j+1, k+1}$, and $\hat{l}_{j+1, k+1} \equiv-l_{j+1, k+1}^{i i} \tau_{i i} /\left(n_{i} m_{i}\right)$ used in Sec. III for calculation of the ion neoclassical transport are given for $j, k=0,1,2,3$ by

$$
\begin{aligned}
& \hat{\mu}_{11}=\sqrt{2}-\ln (1+\sqrt{2}) \simeq 0.53284, \\
& \hat{\mu}_{12}=\frac{1}{2}[4 \sqrt{2}-5 \ln (1+\sqrt{2})] \simeq 0.624993, \\
& \hat{\mu}_{13}=\frac{1}{16}[51 \sqrt{2}-70 \ln (1+\sqrt{2})] \simeq 0.651796, \\
& \hat{\mu}_{14}=\frac{35}{192}[25 \sqrt{2}-36 \ln (1+\sqrt{2})] \simeq 0.66097, \\
& \hat{\mu}_{22}=\frac{1}{8}[39 \sqrt{2}-50 \ln (1+\sqrt{2})] \simeq 1.38571, \\
& \hat{\mu}_{23}=\frac{1}{64}[507 \sqrt{2}-700 \ln (1+\sqrt{2})] \simeq 1.5632, \\
& \hat{\mu}_{24}=\frac{5}{768}[1747 \sqrt{2}-2520 \ln (1+\sqrt{2})] \simeq 1.6248, \\
& \hat{\eta}_{34} \simeq-2.09256, \quad \hat{\eta}_{44} \simeq 3.55606, \\
& \hat{\mu}_{33}=\frac{49}{512}[143 \sqrt{2}-200 \ln (1+\sqrt{2})] \simeq 2.48424, \\
& \hat{\eta}_{11} \simeq 4.04886, \quad \hat{\eta}_{12} \simeq-2.15691, \\
& \hat{\mu}_{34}=\frac{1}{6144}[121837 \sqrt{2}-176400 \ln (1+\sqrt{2})] \simeq 2.73913, \\
& \hat{\eta}_{32} \\
& \left.\hat{\eta}_{14} \simeq 3.2632\right) \\
& \hat{\eta}_{24}
\end{aligned}
$$




$$
\begin{aligned}
& \hat{l}_{22}=\sqrt{2}, \quad \hat{l}_{23}=\frac{3}{4} \sqrt{2}, \quad \hat{l}_{24}=\frac{15}{32} \sqrt{2}, \\
& \hat{l}_{33}=\frac{45}{16} \sqrt{2}, \quad \hat{l}_{34}=\frac{421}{128} \sqrt{2}, \\
& \hat{l}_{44}=\frac{45131}{3072} \sqrt{2},
\end{aligned}
$$

respectively. Here, we should recall that $\hat{l}_{1, k+1}=0$ is derived from collisional momentum conservation and that $\hat{\mu}_{j+1, k+1}$, $\hat{\eta}_{j+1, k+1}$, and $\hat{l}_{j+1, k+1}$ are all symmetric with respect to exchange of $j$ and $k$.

Also, when deriving Eq. (78) in Sec. III, we use the formula for the trapped particles' fraction $f_{t}$ in axisymmetric toroids ${ }^{33}$ with the small inverse aspect ratio $\epsilon \ll 1$,

$$
f_{t}=\frac{3}{\sqrt{2}} \sqrt{\epsilon}\left[1-\int_{0}^{1} \frac{d \kappa}{\kappa^{2}}\left(\frac{\pi}{2 E(\kappa)}-1\right)\right] \simeq 1.46242 \sqrt{\epsilon},
$$

where $E(\kappa)$ denotes the complete elliptic integral of the second kind.

\footnotetext{
${ }^{1}$ M. Wakatani, Stellarator and Heliotron Devices (Oxford University Press, New York, 1998), Chap. 7.

${ }^{2}$ D. E. Hastings, W. A. Houlberg, and K. C. Shaing, Nucl. Fusion 25, 445 (1985).

${ }^{3}$ M. Yokoyama, J. Plasma Fusion Res. 78, 205 (2002).

${ }^{4}$ H. E. Mynick, Phys. Plasmas 13, 058102 (2006).

${ }^{5}$ D. A. Spong, S. P. Hirshman, J. F. Lyon, L. A. Berry, and D. J. Strickler, Nucl. Fusion 45, 918 (2005).

${ }^{6}$ J. N. Talmadge, V. Sakaguchi, F. S. B. Anderson, D. T. Anderson, and A. F. Almagri, Phys. Plasmas 9, 5165 (2001).

${ }^{7}$ G. Grieger, W. Lotz, P. Merkel, J. Nührenberg, J. Sapper, E. Strumberger, H. Wobig, R. Burhenn, V. Erckmenn, U. Gasparino, L. Giannone, H. J. Hartfuss, R. Jaenicke, G. Kühner, H. Ringler, A. Weller, and F. Wagner,
}

Phys. Fluids B 4, 2081 (1992).

${ }^{8}$ H. Sugama and T.-H. Watanabe, Phys. Rev. Lett. 94, 115001 (2005).

${ }^{9}$ H. Sugama and T.-H. Watanabe, Phys. Plasmas 13, 012501 (2006); 14, 079902 (2007)

${ }^{10}$ H. E. Mynick and A. H. Boozer, Phys. Plasmas 14, 072507 (2007).

${ }^{11}$ T.-H. Watanabe, H. Sugama, and S. Ferrando-Margalet, Nucl. Fusion 47, 1383 (2007).

${ }^{12}$ S. Ferrando-Margalet, H. Sugama, and T.-H. Watanabe, Phys. Plasmas 14, 122505 (2007)

${ }^{13}$ W. Zhu, S. A. Sabbagh, R. E. Bell, J. M. Bialek, M. G. Bell, B. P. LeBlanc, S. M. Kaye, F. M. Levinton, J. E. Menard, K. C. Shaing, A. C. Sontag, and H. Yuh, Phys. Rev. Lett. 96, 225002 (2006).

${ }^{14}$ H. Sugama and S. Nishimura, Phys. Plasmas 9, 4637 (2002).

${ }^{15}$ M. Taguchi, Phys. Fluids B 4, 3638 (1992).

${ }^{16}$ S. P. Hirshman, K. C. Shaing, W. I. van Rij, C. O. Beasley, Jr., and E. C. Crume, Phys. Fluids 29, 2951 (1986).

${ }^{17}$ W. I. van Rij and S. P. Hirshman, Phys. Fluids B 1, 563 (1989).

${ }^{18}$ S. Nishimura, H. Sugama, and CHS Group, Fusion Sci. Technol. 46, 77 (2004).

${ }^{19}$ D. A. Spong, Phys. Plasmas 12, 056114 (2005).

${ }^{20}$ C. D. Beidler, M. Yu. Isaev, S. V. Kasilov, W. Kernbichler, G. O. Leitold, H. Maaßberg, S. Murakami, V. V. Nemov, D. A. Spong, V. Tribaldos, in Proceedings of 16th International Stellarator/Heliotron Workshop (National Institute for Fusion Science, Toki, 2007, October 15-19), p. 619.

${ }^{21}$ D. A. Spong (private communication, 2007).

${ }^{22}$ S. R. de Groot and P. Mazur, Nonequilibrium Thermodynamics (NorthHolland, Amsterdam, 1962), Chap. 4.

${ }^{23}$ H. Sugama and W. Horton, Phys. Plasmas 3, 304 (1996).

${ }^{24}$ H. Sugama and W. Horton, J. Plasma Phys. 59, 695 (1998).

${ }^{25}$ A. H. Boozer, Phys. Fluids 26, 1288 (1983).

${ }^{26}$ S. Hamada, Nucl. Fusion 2, 23 (1962).

${ }^{27}$ R. D. Hazeltine and J. D. Meiss, Plasma Confinement (Addison-Wesley, Redwood City, 1992), p. 164.

${ }^{28}$ S. P. Hirshman and D. J. Sigmar, Nucl. Fusion 21, 1079 (1981).

${ }^{29}$ R. Balescu, Transport Processes in Plasmas (North-Holland, Amsterdam, 1988), Vol. 2.

${ }^{30}$ P. Helander and D. J. Sigmar, Collisional Transport in Magnetized Plasmas (Cambridge University Press, Cambridge, 2002).

${ }^{31}$ K. C. Shaing and J. D. Callen, Phys. Fluids 26, 3315 (1983).

${ }^{32}$ S. I. Braginskii, Review of Plasma Physics (Constants Bureau, New York, 1965), Vol. 1, p. 205.

${ }^{33}$ M. N. Rosenbluth, R. D. Hazeltine, and F. L. Hinton, Phys. Fluids 15, 116 (1972). 